\title{
Receptivity and Forced Response to Acoustic Disturbances in High-Speed Boundary Layers
}

\author{
P. Balakumar, Rudolph A. King, Amanda Chou, Lewis R. Owens, and Michael A. Kegerise \\ Flow Physics and Control Branch \\ NASA Langley Research Center, Hampton, VA 23581
}

\begin{abstract}
Supersonic boundary-layer receptivity to freestream acoustic disturbances is investigated by solving the Navier-Stokes equations for Mach 3.5 flow over a sharp flat plate and a 7-deg halfangle cone. The freestream disturbances are generated from a wavy wall placed at the nozzle wall. The freestream acoustic disturbances radiated by the wavy wall are obtained by solving the linearized Euler equations. The results for the flat plate show that instability modes are generated at all the incident angles ranging from zero to highly oblique. However, the receptivity coefficient decreases by about 20 times when the incident angle increases from zero to a highly oblique angle of 68 degrees. The results for the cone show that no instability modes are generated when the acoustic disturbances impinge the cone obliquely. The results show that the perturbations generated inside the boundary layer by the acoustic disturbances are the response of the boundary layer to the external forcing. The amplitude of the forced disturbances inside the boundary layer are about 2.5 times larger than the incoming field for zero azimuthal wavenumber and they are about 1.5 times for large azimuthal wavenumbers.
\end{abstract}

\section{Introduction}

This paper is concerned with the receptivity and the stability of supersonic boundary layers over axisymmetric cones and flat plates at zero degrees angle of attack. It is now understood that transition from a laminar to a turbulent state originates from the internalization of external disturbances into the boundary layer (receptivity) and the exponential growth (linear instability) of these internalized disturbances inside the boundary layer. The second process is governed by the linearized stability equations. The stability characteristics of compressible boundary layers have been thoroughly investigated ${ }^{1}$. The supersonic boundary layers are unstable to three-dimensional disturbances called the first mode. The phase velocity of the unstable first mode disturbances increases from $U_{\infty}-a_{\infty}$ near the lower neutral point to $\mathrm{c}_{\mathrm{s}}$ near the upper neutral point, where $U_{\infty}$ is the freestream velocity, $a_{\infty}$ is the freestream acoustic velocity, and $\mathrm{c}_{\mathrm{s}}$ is the mean velocity at which the generalized inflection point occurs. The unstable region and the amplification rate of the disturbances depend on the flow parameters, Mach number and unit Reynolds number, on the geometry, bluntness and wall condition, and on the frequency and the orientations of the disturbances. However, when these conditions are known, the computations of the boundary layers and the stability characteristics can be performed without much difficulty.

The first process is called receptivity and it depends on several parameters including the flow features near the leading edge, the stability characteristics of the boundary layers, the regions of local inhomogeneity, and the types of external disturbances that impinge on the boundary layer. Since the phase speed of the acoustic disturbances synchronizes with the phase speed of the first modes near the lower branch of the neutral stability curve, it is expected that acoustic disturbances will be very efficient in generating the instability waves in supersonic boundary layers. This observation led to numerous investigations on the interactions of acoustic waves with supersonic boundary layers. The investigations can be divided into two groups. The first group concerns the forced response of parallel and non-parallel boundary layers to freestream acoustic disturbances. ${ }^{2-5}$ In these studies, it was found that acoustic waves excite disturbances inside the boundary layer that are much larger than those in the freestream. The computations also revealed that at some critical Reynolds number the incoming acoustic waves are completely absorbed by the boundary layer. It was postulated that the first mode emerges downstream of this region with the initial amplitude given by the forced response calculations. The second group of investigations are concerned with the generation of the first and the second modes near the leading-edge region due to the interaction with the freestream acoustic disturbances. ${ }^{6-9}$ Fedorov and Khokhlov ${ }^{6}$ investigated the excitation of the first and the second 
modes by the longitudinal acoustic waves using asymptotic theory. They showed that the growing boundary layer diffracts the incoming acoustic wave and generates eigen solutions inside the boundary layer. In a companion paper, ${ }^{7}$ the authors investigated the generation of the instability waves due to incoming acoustic waves at finite angles of incidence. It was identified that at small incident angles the incident and the reflected waves are diffracted by the boundary layer resulting in the formation of the eigen solutions. At large incident angles, the scattered acoustic waves near the leading edge are diffracted by the boundary layer resulting in the generation of the eigen solutions. Later, Fedorov ${ }^{8}$ investigated the receptivity of supersonic boundary layers numerically by imposing a periodic Mach wave near the leading edge of the lower surface of a flat plate. It was shown that the first-mode instability waves were generated on the upper surface of the flat plate and forced responses were created on the lower ssurface.

At high Reynolds numbers, the neutral point is located very close to the leading edge, i.e., within one wavelength of the acoustic wave. The synchronization of the acoustic wave and the first mode occurs even closer. Hence the flow features near the leading edge, such as effects of bluntness, shocks, and non-parallelism, may become very important in the generation of the instability waves. These effects were not included in the aforementioned analysis or computations. In our previous studies, ${ }^{10-12}$ the generation and the evolution of three-dimensional disturbances induced by slow and fast acoustic disturbances and isolated roughness in a supersonic boundary layer over sharp and blunt flat plates and wedges at a freestream Mach number of 3.5 were numerically investigated by solving the full three-dimensional Navier-Stokes equations. It was found that instability waves are generated within one wavelength of the acoustic wave from the leading edge. The wavenumber first decreases from the acoustic value and then slowly increases to the instability wave value, which is smaller than the acoustic wavenumber. The computed receptivity coefficients ${ }^{11}$ for the flow over a sharp flat plate based on the pressure fluctuations at the wall near the neutral points are about 1.20 and 0.07 for the slow and the fast acoustic waves, respectively.

Most of the receptivity analysis and computations are performed for flows over flat plates. Since the mean flow is uniform in the spanwise direction one Fourier mode with constant spanwise wavelength is considered in the analysis. In flows over axisymmetric geometries, the azimuthal wavenumber has to be an integer value due to the circular periodicity in the azimuthal direction. These azimuthal wavenumbers for the unstable waves in supersonic boundary layers are large, on the order of 10 to 30 . When these wavenumbers are converted to wavelengths by dividing the circumferential length by the wavenumber, the wavelengths become very small near the nose and increase gradually in the axial direction. It was found in an earlier investigation ${ }^{10}$ of acoustic receptivity that the instability waves are generated near the leading edge of a flat plate. The question is then how do the acoustic disturbances in the freestream with long spanwise wavelengths generate these small wavelengths or large wavenumber disturbances inside the boundary layer over axisymmetric bodies.

There were several relevant transition experiments performed at the NASA Langley Research Center in the Mach 3.5 Supersonic Low-Disturbance Tunnel (SLDT). Boundary-layer transition data obtained using a rectangular nozzle on a flat plate and on a cone along with freestream noise levels and the power spectral distribution of the freestream noise are presented in Ref. 13. Recently, detailed calibrated hot-wire measurements of the freestream noise characteristics and mass-flux fluctuations inside and at the edge of the boundary layer were made ${ }^{14}$ over a 15 inch long, 7-deg half-angle sharp cone at different unit Reynolds numbers in the SLDT using the axisymmetric nozzle. We will briefly describe the experimental results in the next section. Several intriguing observations were made from the measured data. When the tunnel was operated in quiet mode at a freestream stagnation pressure of 65 psi, the noise level at the boundary layer edge remains below $0.1 \%$ for the first 6 to 7 inches of the cone. Beyond this region, the noise level increases gradually. By the time the flow travels 13 inches down the cone, the noise levels increase to about $0.5 \%$ and $0.2 \%$ for the low-frequency $10 \mathrm{kHz}$ and high frequency $50 \mathrm{kHz}$ disturbances, respectively. The mass-flux fluctuations inside the boundary layer increase from $0.4 \%$ to $3 \%$ and $0.1 \%$ to $1.4 \%$ along the cone from 5 to 13 inches for the low-frequency $10 \mathrm{kHz}$ disturbances and the high-frequency $50 \mathrm{kHz}$ disturbances, respectively. The linear stability theory predicts that the high-frequency $50 \mathrm{kHz}$ disturbances with an azimuthal wavenumber of 30 have the largest integrated growth in this region. Several questions are raised from these experimental observations. First, is the observed growth the local response of the boundary layer to freestream forcing or is it due to the linear stability? Second, why did the high-frequency disturbances not grow exponentially as expected? And third, how does the receptivity vary for the low- and high-frequency freestream disturbances? And finally how does gradually increasing external forcing on the edge of the boundary layer influence the receptivity and the growth of the disturbances inside the boundary layer? Our objective in this paper is to investigate these questions numerically.

As a first step, the computations are performed for the same conditions as in the cone experiment to understand the observed evolution of disturbances inside the boundary layer. For comparison we also performed the simulations for flow over a flat plate at the same conditions. We generated the freestream noise that was radiated from the nozzle walls, by simulating a convecting wavy wall (implemented by blowing and suction at the nozzle 
wall) as the nozzle wall. The acoustic field radiated from this source is obtained by solving the linearized Euler equations using the Fourier transform technique. The computed acoustic field is superimposed on the outer boundary of the computational domain that lies outside the bow shock formed on the cone. Time accurate simulations are performed to investigate the generation and evolution of disturbances inside the boundary layer at different frequencies, convection speeds, and azimuthal wavenumbers.

\section{Experiment}

The experiments were conducted using the Mach 3.5 axisymmetric nozzle in the SLDT. A complete description of the tunnel is given by Beckwith et al. ${ }^{15}$ The test model is a 7-deg half-angle cone that is 15 inches in length with a nominally sharp tip (tip radius $\sim 0.002$ in.). Details on the present experimental setup and hot-wire measurements are given in the paper by King et al. ${ }^{14}$

Boundary-layer stability measurements were acquired over a range of tunnel total pressures from $P_{0} \sim 25$ to 65 psia at a fixed tunnel total temperature of $T_{0}=540 \mathrm{R}$. Most of these measurements were made in a low-noise/quietflow environment with the bleed-valve opened to remove the incoming nozzle wall turbulent boundary layer. Before installing the model, freestream hot-wire measurements were acquired along a vertical centerline plane of the Mach 3.5 axisymmetric nozzle. From the power-spectral density for the mass-flux fluctuations, the variations of the amplitudes along the axial plane for different frequencies were obtained. With the cone model installed in the tunnel, detailed hot-wire measurements were acquired slightly above the boundary layer on the cone and inside the boundary layer. For quiet-flow conditions, data were acquired over the surface of the cone for three unit Reynolds numbers, namely $\operatorname{Re}_{\infty} * 10^{-6} \sim 0.25,0.55$, and $0.65 / \mathrm{in}$. These three unit Reynolds numbers correspond to freestream stagnations pressures of 25,55 , and 65 psi, respectively. The rms (root-mean-square) values are integrated across a $10 \mathrm{kHz}$ bandwidth. Figures 1 and 2 show the variations of the rms massflux along the axial direction for the frequencies of 10 and $50 \mathrm{kHz}$, respectively, for the freestream stagnation pressure of 65 psi. Figure 1 displays the results in a linear-scale and Fig. 2 displays the variation in a log-scale. The figures show the amplitude variation along the axis of the tunnel without the model, the amplitude variation above the boundary layer on the cone, and the maximum amplitude variation inside the boundary layer. The distance, $X_{T}$, is along the centerline of the tunnel measured from the throat. The nozzle throat is located at $X_{T}=0 \mathrm{in}$. and the tip of the cone is located at $X_{T}=18.86 \mathrm{in}$. from the throat. Note that the amplitudes along the centerline without the model are below $0.1 \%$ for $X_{T}<29$ in. for the $10 \mathrm{kHz}$ disturbances, and for $X_{T}<33 \mathrm{in}$. for the $50 \mathrm{kHz}$ disturbances. The disturbances grow slowly beyond these points. The amplitude for $f=10 \mathrm{kHz}$ reaches a value of $0.25 \%$ at $X_{T} \sim 32 \mathrm{in}$. and the amplitude for the $f=50$ $\mathrm{kHz}$ reaches a value of $0.13 \%$ at $X_{T} \sim 34 \mathrm{in}$. At the boundary-layer edge, the amplitude of the low-frequency disturbances, $f=10 \mathrm{kHz}$, remains below $0.1 \%$ up to $X_{T}=26$ in. and grows slowly downstream before reaching a value of $0.5 \%$ at $X_{T} \sim 30 \mathrm{in}$. The amplitude for the high-frequency $f=50 \mathrm{kHz}$ remains below $0.1 \%$ up to $X_{T} \sim 29 \mathrm{in}$. and reaches a value of $0.2 \%$ at $X_{T} \sim 31$ inches. The maximum mass-flux fluctuations inside the boundary layer for the low-frequency $f=10 \mathrm{kHz}$ wave is about $0.4 \%$ at $X_{T} \sim 24 \mathrm{in}$. and grows downstream before reaching a value of $3 \%$ at $X_{T} \sim 30$ inches. The maximum mass-flux fluctuations for the high-frequency $f=50 \mathrm{kHz}$ wave is about $0.1 \%$ at $X_{T} \sim 24$ in. and is about $1.4 \%$ at $X_{T} \sim 31$ inches. From $X_{T}=24$ to 30 inches, the low frequency wave grew by approximately a factor of 7 and the high-frequency wave grew by approximately a factor of 14 .

Figure 3 shows the N-Factor variation obtained from the linear stability computations for different frequencies $f$, and azimuthal wavenumbers, $m$. In Fig. $3 b$, it is seen that the frequency and the azimuthal wavenumber of the most amplified wave at $X \sim 12 \mathrm{in}$. from the nose tip is about $f=50 \mathrm{kHz}$ and $m=30$. Here $X$ is measured from the apex of the cone. The maximum N-Factor at this location is about 8.5. The maximum N-Factor obtained for the lowfrequency wave with $f=10 \mathrm{kHz}$ at this location is about 1.4 . The linear stability computations predict that the highfrequency disturbances will be amplified by about 5000 times from the neutral point $X_{N}=1.7$ to $X=12 \mathrm{in}$., and the low-frequency disturbances will be amplified by about 4 times. The experiment did not show the explosive growth indicated by the computations for the high-frequency disturbances. In Fig. 2, we plotted the linear amplitude growth curve obtained from the simple N-Factor calculations. It is seen that growth of the low-frequency wave follows the linear stability results closely, but the growth of the high-frequency disturbances differs considerably from the predicted curve.

\section{Formulation and Flow Conditions}

We consider supersonic flow over a flat-plate and an axisymmetric 7-deg half-angle cone at zero angle of attack at freestream Mach numbers of 3.5. The flat plate has a sharp leading edge with a half-thickness of 0.00125 inches and the cone has a nose radius of 0.002 inches. The flat plate is placed along the axis of a two-dimensional nozzle and the cone is placed along the axis of a cylindrical nozzle as shown in Fig. 4. The detailed experiments were 
performed only for the flow over the cone in the SLDT. ${ }^{14}$ The computational setups are shown in Figs. 4(a) and (b) for the flat plate and the cone, respectively. The freestream acoustic field is generated by placing harmonically oscillating blowing and suction sources at the surface of the nozzles. For the flat-plate configuration, noise is radiated symmetrically from the top and bottom walls of the two-dimensional nozzle. For the cone configuration, noise is radiated from an axisymmetric nozzle. The noise field radiated from the harmonic sources is obtained by solving the linearized Euler equations using the Fourier transform method. The computed acoustic field is superimposed on the outer boundary of the computational domain that lies outside the bow shock formed on the flat plate and the cone. The flow parameters used in the computations are the same as in the experiment performed for the cone and are given below.

$\begin{array}{ll}\text { Freestream Mach number } & 3.5 \\ \text { Freestream stagnation pressure } & 65 \mathrm{psia} \\ \text { Freestream Reynolds number } & 0.65^{*} 10^{6} / \text { in } \\ \text { Freestream temperature } & 156^{\circ} \mathrm{R}\end{array}$

The wall temperature condition is prescribed as a constant adiabatic temperature $\left(483^{\circ} \mathrm{R}\right)$ near the nose tip $(X<2$ in.) and is gradually followed by a linear wall temperature distribution that increases to $502^{\circ} \mathrm{R}$ at $X=12 \mathrm{in}$. The conditions downstream of the shock on the surface of the cone obtained from solving the Taylor-Maccoll equations are:

$\begin{array}{cl}\text { Shock angle } & =17.66 \mathrm{deg} \\ M_{s} & =3.27 \\ U_{s} & =1.18 U_{\infty} \\ T_{s} & =1.10 T_{\infty} \\ \rho_{s} & =1.27 \rho_{\infty} \\ p_{s} & =1.39 p_{\infty} \\ R e_{s} / i n & =1.124^{*} 10^{6}\end{array}$

Here, $M_{s}, U_{s}, T_{s}, \rho_{s}$ and $p_{s}$ are the inviscid surface Mach number, velocity, temperature, density, and the pressure, respectively. The unit Reynolds number is defined based on the surface conditions, $R e_{s}=U_{s} / v_{s}$ where $v_{s}$ is the kinematic viscosity at the surface.

\section{Governing Equations}

The equations solved are the three-dimensional unsteady compressible Navier-Stokes equations in conservation form in Cartesean and cylindrical coordinates for the flat plate and cone, respectively. The governing equations are given in earlier papers. ${ }^{10,11}$ The viscosity, $\mu$, is computed using Sutherland's law and the thermal conductivity, $k$, is given in terms of the Prandtl number, $P r$. The flow variables are non-dimensionalized by their corresponding reference variables in the freestream. For the computations, the equations were transformed from the physical coordinate system $(x, y, z)$ or $(x, r, \theta)$ to the computational curvilinear coordinate system $(\xi, \eta, \zeta)$ while preserving conservative formulation.

\section{Solution Algorithm}

The governing equations were solved using a $5^{\text {th }}$-order accurate weighted essentially non-oscillatory (WENO) scheme for space discretization and a $3^{\text {rd }}$-order total-variation-diminishing (TVD) Runge-Kutta scheme for time integration. The WENO and TVD methods and formulas are explained in Shu. ${ }^{16}$ The application of the ENO method to the Navier-Stokes equations is presented by Atkins. ${ }^{17}$ The solution method implemented in the present computations is described in Balakumar et al. ${ }^{18}$

A schematic diagram of the computational setup is shown in Fig. 4. The outer boundary of the computational domain lies outside the shock and follows a parabola so that the boundary-layer growth is accurately captured. At the outflow boundary of the computational domain, an extrapolation boundary condition was used. The simulations employed viscous conditions for the velocities and the measured temperature distribution for the wall temperature. Symmetric conditions were employed in the spanwise direction. The density was computed from the continuity 
equation. In the mean-flow computations, the freestream values at the outer boundary were prescribed. In all cases, the model centerline was aligned with the freestream flow. In the unsteady computations, acoustic perturbations were superimposed on the uniform mean flow at the outer boundary of the computational domain.

The solution procedure began by computing the steady mean flow. This was achieved by performing unsteady computations using a variable time step until the maximum residual reached a small value $\left(\sim 10^{-11}\right)$. For those computations, a CFL number of 0.2 was used. The next steps were (1) to introduce unsteady acoustic disturbances at the outer boundary of the computational domain and (2) to perform time-accurate simulations to investigate the interaction of these disturbances with the boundary layer and their subsequent downstream evolution. Linearized Euler equations in a uniform mean flow or the linearized Navier-Stokes equations in a similarity boundary layer were solved to obtain analytical expressions for the acoustic disturbances that were to be superimposed at the outer boundary of the computational domain.

The computational grid, which was generated using analytical formulae, stretches in the normal, $\eta$, direction close to the wall and is uniform outside of the boundary layer. In the $\xi$ direction, the grid was very fine near the nose and then uniform in the region downstream. Since we have to transmit oblique low- and high-frequency acoustic disturbances from the outer boundary toward the flat plate and the cone very fine grids were used in the normal direction. For the low-frequency simulations 751 points were used in the normal direction and 1501 points in the high-frequency simulations. We always maintained about 30 points per wavelength in the streamwsie direction. We used 11 points per half wavelength in the spanwise or the azimuthal direction.

\section{Results}

\section{A. Mean flow}

In Fig. 5, the mean-flow density contours and the boundary-layer profiles computed using the WENO code for the flow over a smooth plate are shown. Figure 5(a) shows the density contours in the entire computational domain. The half thickness of the plate is $b=0.00125$ inches. It is seen that very close to the leading edge, there exists a strong shock. This compression expands over the leading edge, and the shock becomes weaker away from the nose region. The outer boundary of the leading-edge shock is located approximately 0.00078 inches $(0.6 \mathrm{~b})$ upstream of the leading edge. Figure 5(b) shows the boundary-layer profiles for the mass-flux quantity at $X=1,4,8$, and 12 inches from the leading edge. Similarly, Figs. $6(a, b)$ depict the mean density contours and the mass-flux boundarylayer profiles for the flow over the cone. The cone has a nose radius of $r_{0}=0.002$ inches. The leading-edge shock is located approximately 0.0004 inches $\left(0.2 \mathrm{r}_{0}\right)$ upstream of the nose. The computed boundary-layer profiles agree very well with the experimental profiles as shown in Ref. 14.

\section{B. Acoustic Field Radiated from the Nozzle Wall}

\section{(1) Plane Acoustic Field}

In previous studies, we investigated the interactions of a plane acoustic field with flat plates, wedges, and cones at supersonic and hypersonic Mach numbers. ${ }^{10-12}$ The solutions of the acoustic field are obtained by solving the linearized Euler equations in a uniform flow. The governing equations and the solutions for acoustic waves in Cartesian and cylindrical coordinate systems are given in Ref. 19. The acoustic pressure $p$ in a Cartesian coordinate system is in the form

$$
p_{a c}=\tilde{p}_{a c} e^{i\left(\alpha_{a c} x+\varepsilon_{a c} y+\beta_{a c} z-\omega t\right)}
$$

Here $\alpha_{a c}, \beta_{a c}, \varepsilon_{a c}$ are the acoustic wavenumbers, and $\omega$ is the frequency of the acoustic disturbance. The wavenumber in the $y$-direction $\varepsilon_{a c}$ determines the incident angle of the acoustic waves. The dispersion relation among the acoustic wavenumbers $\alpha_{a c}, \beta_{a c}, \varepsilon_{a c}$ and the frequency $\omega$ is given by

$$
\left(\alpha_{a c} U_{\infty}-\omega\right)^{2}=\left(\alpha_{a c}^{2}+\beta_{a c}^{2}+\varepsilon_{a c}^{2}\right) a_{\infty}^{2}
$$


The incident angle $\theta_{y}$ is defined as $\theta_{y}=\tan ^{-1}\left(\frac{\varepsilon_{a c}}{\alpha_{a c}}\right)$ and the convective speed in the $x$-direction is defined as $C=\frac{\omega}{\alpha_{a c}}$. The incident angle is the angle made by the constant phase lines with the $y$-axis. Similarly, the acoustic field in the cylindrical coordinate system takes the following form

$$
p_{a c}=\tilde{p}_{a c} J_{m}\left(\alpha_{n} r\right) e^{i\left(\alpha_{a c} x+m \theta-\omega t\right)} .
$$

where, $J_{m}$ is the Bessel function of the first kind of order $m$. The dispersion relation among the wavenumber $\alpha_{a c}, \alpha_{n}$ and the frequency $\omega$ is given by

$$
a_{\infty}^{2} \alpha_{n}^{2}=\left(\alpha_{a c} U_{\infty}-\omega\right)^{2}-\alpha_{a c}^{2} a_{\infty}^{2} .
$$

We computed the incident angle, wavelength, and the phase speed for the plane and circular acoustic fields and compared them with the results obtained from the linear stability theory. In Table 1, the acoustic wavelengths for different phase speeds corresponding to different incident angles, are compared with the wavelengths of the instability waves at the neutral points for the flat plate and cone boundary layers. The frequency and the spanwise wavelength of the most amplified instability wave on the flat plate are $20 \mathrm{kHz}$ and 0.30 inches, respectively. The wavelength of the instability wave at the neutral point is about 0.72 inches. The wavelength of the acoustic wave decreases from 0.535 to 0.402 inches when the incident angle increases from 0 to 60 degrees. This suggests that when the incident angle increases, the synchronization between the acoustic waves and the instability waves becomes weaker. In Fig. 7(a), we plot the variation of the wavelength of the instability wave along the plate. We also marked by blue lines the wavelengths of the acoustic waves at different incident angles. It is seen that the wavelength of the instability wave increases steeply near the leading edge region of the plate and increases gradually further downstream. Hence it is expected based on the computations that the receptivity, i.e., the generation of instability waves inside the boundary layer by the freestream disturbances, will be strong only near the leading-edge region of a flat plate.

The wavelengths of the acoustic waves and the wavelengths of the instability waves at the neutral points for the cone boundary layer are provided in Table 1 . We present the results for a low, $10 \mathrm{kHz}$, and a high, $50 \mathrm{kHz}$, frequency at an azimuthal wave number of $m=30$. It is to be noted that the frequency and the azimuthal wavenumber of the most amplified instability wave are $f=50 \mathrm{kHz}$ and $m=30$. The wavelengths at the neutral points are about 1.29 and 0.37 inches for the 10 and $50 \mathrm{kHz}$, respectively. The wavelengths of the acoustic waves decrease from 1.54 to 0.51 inches for the low-frequency wave and decrease from 0.31 to 0.10 inches for the high-frequency wave when the convective speeds decrease from 0.6 to 0.2 . The corresponding incident angles increase from 30 to 68 degrees. Hence waves incoming at shallow angles could synchronize with instability waves more efficiently than the highly oblique waves. Another important observation is that the acoustic field in the radial direction is determined by the Bessel function of order m, Eq. (3). It is known that the Bessel function decays like $r^{m}$ as $r$ approaches zero. Hence, the nose region of the cone will not be directly influenced by the radiated acoustic field with large azimuthal wavenumber from the nozzle wall. In Figs. 7(b) and (c), we plot the variation of the wavelength of the instability waves for $50 \mathrm{kHz}$ and $10 \mathrm{kHz}$ with $m=30$, respectively. In contrast to the flat-plate case, the wavelength of the instability waves first decreases near the nose region and very slowly increases further downstream. We also marked the wavelengths of the acoustic waves at different convection speeds in the figure. It is seen that for the high frequency, $f=50 \mathrm{kHz}$, only the acoustic disturbances that are convecting at high speeds $c \sim$ $0.6 U_{\infty}$ have comparable wavelengths to the instability waves. At lower frequencies, the acoustic waves convecting at high speeds have wavelengths comparable to the instability waves near the nose region. 
$46^{\text {th }}$ AIAA Fluid Dynamics Conference, June 13-17, 2016, Washington D.C

Table 1 Values of $\alpha_{a c}$ and wavelength for flat plate and cone at different incident angles.

\begin{tabular}{|c|c|c|c|c|c|c|}
\hline & $\mathbf{f}(\mathbf{k H z})$ & $\lambda_{\mathbf{z}}$ (in.) & $\boldsymbol{\theta}_{\boldsymbol{y}}$ (deg.) & $\begin{array}{c}\text { Phase } \\
\text { speed } \\
\text { or } \mathbf{~}\end{array}$ & $\boldsymbol{\lambda}_{\text {ac, } \mathbf{x}}$ (in.) & $\begin{array}{c}\boldsymbol{\lambda}_{\mathbf{x}} \text { (in.) } \\
\text { Neutral }\end{array}$ \\
\hline \multirow{3}{*}{$\begin{array}{c}\text { Flat } \\
\text { plate }\end{array}$} & 20 & 0.30 & 0 & 0.41 & 0.535 & 0.722 \\
\cline { 2 - 7 } & & & 30 & 0.40 & 0.520 & \\
\cline { 2 - 7 } & & & 60 & 0.25 & 0.402 & \\
\hline \multirow{3}{*}{ Cone } & 10 & 30 & 30 & 0.60 & 1.545 & 1.291 \\
\cline { 2 - 7 } & & & 40 & 0.40 & 1.030 & \\
\cline { 2 - 7 } & & & 68 & 0.20 & 0.515 & \\
\cline { 2 - 7 } & 50 & 30 & 30 & 0.60 & 0.309 & 0.371 \\
\cline { 2 - 7 } & & & 40 & 0.40 & 0.206 & \\
\cline { 2 - 7 } & & & 68 & 0.20 & 0.103 & \\
\hline
\end{tabular}

\section{(2) Nonuniform Acoustic Field}

The acoustic field radiated from the nozzle wall is, in general, not uniform in the axial direction. Initially, the nozzle wall remains laminar and the radiated noise level remains very small or undetectable with current measurement techniques. The nozzle wall boundary layers transition to turbulence, and the noise level increases gradually along the axial direction. This was observed in the experiment ${ }^{14}$ as shown in Fig. (1). The measurements in the freestream showed that as the nozzle-wall flow transitions to turbulence the source speed in the nozzle boundary layer is around 0.2 times the freestream speed. This is based on the broadband mass flux. We modeled the radiated noise field with increasing amplitude by placing a convecting wavy wall on the surface of the nozzle. The amplitude of the wavy wall is increasing in the axial direction, but the convecting velocity is kept at a constant value. The normal velocity imposed at the nozzle wall takes the following form.

In the two-dimensional nozzle:

$$
V_{0}(x, H, z, t)=\frac{V_{\max }}{2}\left\{\tanh \frac{x-x_{1}}{\Delta_{1}}-\tanh \frac{x-x_{2}}{\Delta_{2}}\right\} \cos \left(\alpha_{a c} x-\omega t\right) \cos (\beta z)
$$

In the axisymmetric nozzle:

$$
V_{0}\left(x, r_{0}, \theta, t\right)=\frac{V_{\max }}{2}\left\{\tanh \frac{x-x_{1}}{\Delta_{1}}-\tanh \frac{x-x_{2}}{\Delta_{2}}\right\} \cos \left(\alpha_{a c} x-\omega t\right) \cos (m \theta)
$$

Here $V_{0}(x, y, z, t)$ and $V_{0}(x, r, \theta, t)$ are the normal velocities imposed as the boundary conditions on the nozzle walls located at the heights $y=H$ and $r=r_{0}$, respectively. The coordinate $x_{l}$ determines the location along the nozzle at which the amplitude of the source starts to increase from zero value and the coordinate $x_{2}$ determines the location at which the amplitude starts to decrease again to zero. The parameters $\Delta_{l}$ and $\Delta_{2}$ determine the region and the rate of growth of the noise levels. The acoustic field radiated from the source is computed by solving the linearized Euler equations using Fourier transform methods. The solutions for the acoustic pressure field are given by the following expressions.

In the two-dimensional nozzle:

$$
p(x, y, z, t)=\operatorname{Real}\left\{e^{-i \omega t} \int_{-\infty}^{+\infty} \gamma M^{2} \tilde{V}\left(k_{x}\right) \frac{\left(k_{x}-\omega\right) e^{\lambda y}}{i \lambda e^{\lambda H}} e^{i k_{x} x} d k_{x}\right\} \cos (\beta z)
$$


where

$$
\lambda^{2}=-\left\{M^{2}\left(k_{x}-\omega\right)^{2}-k_{x}^{2}-\beta^{2}\right\}
$$

and $\gamma$ is specific gas ratio.

In the axisymmetric nozzle:

$$
p(x, r, \theta, t)=\operatorname{Real}\left\{e^{-i \omega t} \int_{-\infty}^{+\infty} \gamma M^{2} \tilde{V}\left(k_{x}\right) \frac{\left(k_{x}-\omega\right) J_{m}(\lambda r)}{i \lambda J_{m}^{\prime}\left(\lambda r_{0}\right)} e^{i k_{x} x} d k_{x}\right\} \cos (m \theta)
$$

where

$$
\lambda^{2}=\left\{M^{2}\left(k_{x}-\omega\right)^{2}-k_{x}^{2}\right\}
$$

Here $\tilde{V}\left(k_{x}\right)$ is the Fourier transform of the normal velocity $V_{0}, J_{m}$ is the Bessel function of order $m$, and $J_{m}{ }^{\prime}$ is its derivative. These integrals are evaluated numerically to obtain the acoustic field radiated from a nonuniform moving source. We also performed direct numerical simulations (DNS) by solving the Euler equations using the same numerical technique. The results from the Fourier transform method and the DNS agree very well. Figure 8 shows the radiated pressure field from the axisymmetric nozzle wall obtained using the transform method. The results are shown for a frequency of $f=10 \mathrm{kHz}$ at three azimuthal wavenumbers $m=0,10$, and 30 . The results are shown for the case when the source is convecting at a speed of 0.2 times the freestream velocity. We also drew the 7-deg halfangle cone in these figures. The constant phase lines are inclined at about 68 degrees to the vertical axis. For axisymmetric disturbances with $m=0$, as expected, the acoustic field extends to the center of the nozzle. With increasing azimuthal wavenumbers, we note that there exists a quiet zone in the center of the nozzle. This quiet zone increases in height with increasing azimuthal wavenumber. In Figs. (9a, b), we plot the radiated acoustic pressure field along the surface of the cone. We also included the pressure field along the surface of the nozzle. In Fig. 9(a), we show the instantaneous pressure field, and in Fig. 9(b), we show the variation of the amplitude at different azimuthal wavenumbers $m=0,10$, and 30. As we discussed earlier, the amplitude of the pressure field becomes smaller in the nose region of the cone as the azimuthal wavenumber increases. The amplitude on the cone reaches a value of about $10^{-6}$ at $x=0,2$, and 6.5 inches for $m=0,10$, and 30, respectively. Figure 10 similarly depicts the amplitude variations for the frequency $f=50 \mathrm{kHz}$ at different azimuthal wavenumbers $m=0,10$, and 30 . In this case, the amplitude on the cone reaches a value of about $10^{-6}$ at $x=0,0.5$, and 1.5 inches for $m=0,10$, and 30, respectively. It shows that the extent of the quiet zone decreases with increasing frequency. It implies that even though disturbances with large azimuthal wavenumbers are the most unstable disturbances according to linear theory, the external disturbances with large azimuthal wavenumbers have negligible amplitudes on the boundary layer edge in the early part of the cone. In a later section, we will investigate how this external acoustic field interacts with the cone boundary layer, and what kinds of disturbances are generated inside the boundary layer.

\section{Disturbance Field Inside the Boundary Layer}

The unsteady flow field in a boundary layer consists of all the discrete eigen solutions and the continuous spectrum, which appears because the domain is unbounded and the linearized stability equations admit solutions that are bounded at infinity. The details about the derivation and the computation of the discrete and the continuous spectrum are given in Ref. 20. For a supersonic boundary layer, there exist seven branches of the continuous spectrum in the complex wavenumber space. Two of them are the fast and slow moving acoustic waves. Hence the freestream acoustic disturbances enter the boundary layer through the continuous spectrum. The eigenfunction corresponding to the continuous spectrum is obtained by solving the linearized stability equations with an inhomogeneous boundary condition at the far field. Keeping only the bounded solution at the far field and discarding the exponentially growing solution, the solution in the far field corresponding to the acoustic wave continuous spectrum takes the form

$$
\left\{u, v, w, T, p, u^{\prime}, w^{\prime}, T^{\prime}\right\}^{T}=C_{1} q_{1} e^{-\mu_{1} y}+C_{2} q_{2} e^{-\mu_{2} y}+C_{3} q_{3} e^{-\mu_{3} y}+C_{4} q_{4} e^{-i \mu y}+C_{5} q_{5} e^{+i \mu y}
$$


The first three terms on the right hand side represent the exponentially decaying solution and the last two terms represent the oscillatory acoustic disturbances. At high Reynolds numbers the wavenumber in the $y$-direction, $\mu$, is given by the acoustic dispersion relation.

$$
\mu^{2}=M^{2}\left(k_{x}-\omega\right)^{2}-k_{x}^{2}-\beta^{2}
$$

For the last two terms in Eq. 11, the first term is the incident acoustic wave and the second term is the reflected acoustic wave. Hence by fixing the amplitude of the incident wave, for example by setting the amplitude of the pressure of the incoming wave $C_{4} q_{45}=p_{0}$, the coefficient $C_{4}$ can be fixed. We performed the computations for the flat-plate and cone similarity boundary layers at several incident angles. The incident angle is given by $\tan ^{-1}\left(\mu / k_{x}\right)$. Figures 11-13 show the distribution of the amplitudes of the mass-flux fluctuations versus the Blasius similarity coordinate inside the boundary layer obtained by solving the inhomogeneous problem with the acoustic forcing at the freestream. Figure 11 depicts the results for the flow over a flat plate for a frequency of $f=20$ $\mathrm{kHz}$ and a spanwise wavelength of $\lambda_{z}=0.3$ inches at different incident angles of 5,10,30, and 65 degrees. The results are shown for a Reynolds number based on the similarity length scale of $R e_{x}=\sqrt{U_{e} x / v_{e}}=2270$. Here, $U_{e}$ and $v_{e}$ are the mean velocity and kinematic viscosity at the boundary-layer edge, respectively. This corresponds to $x$ $=7$ inches for the freestream parameters used in the experiment and in the simulations. We also plot the eigenfunction distribution obtained from the linear stability theory for comparison. It is seen that response of the boundary layer to freestream forcing increases gradually with increasing incident angles. The maximum amplitude inside the boundary layer occurs at a high incident angle of 65 degrees. The maximum amplitude inside the boundary layer is about 0.9 times the maximum amplitude in the freestream at this incident angle of 65 degrees. We note that the disturbance amplitudes oscillate in the freestream. We select the first maximum amplitude in the freestream to compare with the amplitudes inside the boundary layer. Comparing the eigenfunctions distributions obtained from the linear stability theory and the freestream acoustic forcing, we observe that with the forcing the distribution has a two-peak structure compared to one peak for the linear stability theory. The eigenfunction from the linear stability peaks near $\eta=7.3$ and the eigenfunction from the forcing peaks at a slightly higher height, $\eta=$ 8.4 .

Similarly, Figures 12 and 13 show the results for the cone similarity boundary layers at frequencies of 10 and 50 $\mathrm{kHz}$, respectively. The results are shown for azimuthal wavenumbers of $m=0$ and 30 . We use different scales in the abscissa for clarity. The results are shown for an $R e_{x}==2425$. This corresponds to $x=8$ inches for the freestream parameters used in the experiment and in the simulations. We also plotted the eigenfunction distribution obtained from the linear stability theory for comparison. The results for $f=10 \mathrm{kHz}$ and $m=0$, Fig. 12a, show that the response of the forcing is stronger in the cone boundary layer than in the flat-plate boundary layer. The response to the forcing increases gradually with the incident angle up to 30 degrees and remains almost the same with further increase. The ratio between the maximum amplitudes inside and outside the boundary layer is about 5.2 for an incident angle of 30 degrees. This ratio is about 4 for the incident angle of 65 degrees. Comparing the eigenfunction distributions obtained from the linear stability theory and the freestream acoustic forcing, we observe that the shapes appear similar in both cases with the peak occurring slightly higher for the eigenfunction from the linear stability theory. In the lower part of the boundary layer, the eigenfunction from the forcing has larger amplitude than that from the linear stability theory. The results for the higher azimuthal wavenumber of $m=30$, Fig. 12(b), show that the response increases gradually with the incident angle and peaks at an incident angle of 65 degrees. The ratio between the maximum amplitudes inside and outside the boundary layer is about 1.4 for the incident angle of 65 degrees. This is about 4 times smaller than at the azimuthal wavenumber of $m=0$. The maximum amplitudes of the eigenfunctions obtained from the forcing and the linear stability theory occur at the same location $\eta=4.8$, but the shapes are different in the lower part of the boundary layer. In the lower part of the boundary layer, the eigenfunction from the forcing display an inflectional type profile with larger amplitudes compared to a smooth profile with lower amplitude for the eigenfunction from the linear stability theory (LST).

Figures 13(a) and (b) display the eigenfunctions obtained from the forcing and the linear stability theory for the higher frequency of $f=50 \mathrm{kHz}$ at azimuthal wavenumbers of $m=0$ and 30 , respectively. The Reynolds number is the same as for the $f=10 \mathrm{kHz}$ case. In the two-dimensional case for $m=0$, the response increases gradually up to an incident angle of 30 degrees and decreases with further increase in incident angle. The ratio between the maximum amplitudes inside and outside the boundary layer is about 2.5 for the incident angle of 30 degrees. The shapes of the eigenfunctions appear similar in both cases and the eigenfunctions peak near $\eta=4.8$. In the larger azimuthal 
wavenumber case for $m=30$, the amplitudes with the forcing increase gradually up to an incident angle of 30 degrees and decrease with further increase in the incident angle. The ratio between the maximum amplitudes inside and outside of the boundary layer is about 1.0 in this case for the incident angle of 30 degrees. The eigenfunctions with the forcing display a double-peak structure compared to the one-peak structure for the eigenfunction from linear stability theory. The eigenfunction from the linear stability peaks near $\eta=4.3$ and the eigenfunction from the forcing peaks at a slightly higher height of $\eta=4.9$.

\section{Interaction of Three-Dimensional Acoustic Disturbances with a Flat Plate}

We first investigate the interaction of uniform and nonuniform three-dimensional acoustic disturbances impinging at different incident angles on a sharp flat plate. We performed three simulations to understand the receptivity process in supersonic flows over a flat plate placed in a two-dimensional nozzle. First, we consider the interaction of three-dimensional plane acoustic waves with uniform amplitude impinging at zero incidence angle with the flat plate. Next, we simulate the interaction of three-dimensional acoustic waves with uniform amplitude impinging the flat plate at a highly oblique angle of 68 degrees. This corresponds to acoustic disturbances convecting at a speed of 0.2 times the freestream velocity. Finally, we investigate the interaction of a nonuniform three-dimensional acoustic field impinging on the flat plate at a highly oblique angle of 68 degrees. In all cases, the frequency of the disturbances is $f=20 \mathrm{kHz}$ and the spanwise wavelength is 0.3 inches. These parameters give the largest N-Factor of 6.0 near $x=14$ inches, Fig. 3(a). In the first two cases, the symmetric acoustic field that impinges on the outer boundary is taken to be in the following form.

$$
\begin{aligned}
p_{a c}^{\prime}= & \operatorname{Real}\left\{\tilde{p}_{a c} e^{i \alpha_{a c} x \pm i \beta z+i \varepsilon_{a c} y-i \omega t}\right\} \\
& +\operatorname{Real}\left\{\tilde{p}_{a c} e^{i \alpha_{a c} x \pm i \beta z-i \varepsilon_{a c} y-i \omega t}\right\} .
\end{aligned}
$$

\section{Interaction of Uniform Acoustic Waves with a Flat Plate}

Three-dimensional acoustic disturbances with uniform amplitudes are superimposed at the outer boundaries, and time accurate simulations are performed. The amplitude of these forced acoustic disturbances was given a small value of $\tilde{p}_{a c} / p_{\infty}=1 * 10^{-5}$ to ensure that the disturbances evolving in the boundary layer remained in the linear regime. First, we present the results for an incidence angle of 0 degrees $\left(\varepsilon_{a c}=0\right)$. We have performed similar computations previously ${ }^{10}$ at a higher unit Reynolds numbers of $0.65^{*} 10^{6} /$ in. Figure 14(a) shows the results for the evolution of the unsteady mass-flux fluctuations obtained from the simulation at a fixed time.

Figure 14(b) shows the instantaneous maximum mass-flux fluctuations along the flat plate on a log scale. Figure 14(b) also includes the results from the linear parabolized stability equations (PSE) computations obtained for the same mean boundary-layer profiles. We also plot the mass-flux fluctuations outside the boundary layer in this figure. The conclusions are very similar to the results presented in the previous study. ${ }^{10}$ The figure clearly shows the initial generation and the eventual exponential growth of the instability waves inside the boundary layer. The acoustic waves with wavelengths that are closer to the wavelength of the instability waves are transformed smoothly into instability waves near the leading-edge region and grow exponentially downstream. The growth of the disturbances agrees very well with the PSE results about one acoustic wavelength downstream of the neutral point. Following the PSE results up to the neutral point, the initial amplitude of the instability waves at the neutral point can be estimated. The neutral point is located approximately at $x=0.66$ inches from the leading edge of the plate. From these values, the receptivity coefficients can be evaluated. Specifically, the receptivity coefficient $C_{\text {recpt }, p_{\text {wall }}}$ is defined by the initial amplitude of the pressure fluctuations at the wall at the neutral point non-dimensionalised by the freestream acoustic pressure, i.e.,

$$
C_{\text {recpt }, p_{\text {wall }}}=\frac{\tilde{p}_{\text {wall }}}{\tilde{p}_{a c}}
$$

The computed receptivity coefficient is

$$
C_{\text {recpt, }, p_{\text {wall }}}=0.50
$$


Similarly, the receptivity coefficients based on the maximum mass-flux fluctuations inside the boundary layer normalized by $(\rho u)_{a c}$ is calculated.

$$
C_{\text {recpt }, \text { pu } \text { max }_{\text {max }}}=6.40
$$

Next, we consider the interaction of uniform acoustic disturbances inclined at 68 degrees with the flat plate. Figure 15(a) shows the results for the evolution of the unsteady mass-flux fluctuations obtained from the simulation at a fixed time. The acoustic disturbances are impinging the plate symmetrically from the bottom and top of the plate. We observe that across the shock only the acoustic disturbances impinging from the top are transmitted through the shock. Then these disturbances interact with the boundary layer and the plate and are reflected back into the inviscid region. Figure 15(b) shows the instantaneous maximum mass-flux fluctuations along the plate in a $\log$ scale. Figure 15(b) also includes the results from the PSE computations obtained for the same mean boundary-layer profiles. The wavelength of the acoustic disturbances in the streamwise direction is about 0.26 inches and the wavelength of the instability wave near the neutral point is about 0.72 inches. Starting from $x=4$ inches, we can discern the instability waves clearly, and the PSE and the DNS results also agree very well. In the leading-edge region up to $x=4$ inches, we observe the short wavelength disturbances inside the boundary layer. These disturbances are due to the forcing by the short wavelength acoustic disturbances. To separate the forcing and the eigen solutions, we need to perform a modal analysis using an adjoint method. ${ }^{21}$ We followed the PSE solutions up to the neutral point to extract the receptivity coefficients in this case. The computed receptivity coefficients are

$$
\begin{aligned}
& C_{\text {recpt }, p_{\text {wall }}}=0.024 \\
& C_{\text {recpt }, \rho u_{\text {max }}}=0.34
\end{aligned}
$$

The receptivity coefficients are about 20 times smaller than that for the previous case. Hence, in high-speed tunnels where the radiated noise impinges the plates at high incidence angles, the initial amplitudes of the disturbances will be much smaller than those generated by a plane wave at zero incident angles.

\section{Interaction of Nonuniform Acoustic Waves with a Flat Plate}

We simulated the interaction of nonuniform acoustic disturbances incident at 68 degrees with the flat plate. The convecting speed of the eddy is $0.2 U_{\infty}$. The amplitude of the mass-flux fluctuations outside the boundary layer increases from $1 * 10^{-6}$ near the leading edge to $6^{*} 10^{-5}$ near the end of the plate at $x=15$ inches. Figure 16(a) depicts the contours of the mass-flux fluctuations at a fixed time. Figure 16(b) shows the instantaneous maximum mass-flux fluctuations along the cone on a log scale. Figure 16(b) also includes the results from the PSE computations. We also plot the mass-flux fluctuations outside the boundary layer in this figure. Again the wavelengths of the external disturbances are on the order of 0.26 inches. The results obtained from the simulation follow the PSE results from $x$ $=7$ inches. Even in this region, comparing with Figs. 14(b) and 15(b), one sees small modulations in the mass-flux amplitude variation along the axis. These modulations are due to the forcing by the acoustic disturbances as these disturbances are comparable to the instability disturbances. As we mentioned earlier, a modal analysis will shed more information about the origin of the instability waves. We followed the PSE solutions up to the neutral point to extract the receptivity coefficient in this case. The computed receptivity coefficient based on the maximum mass flux fluctuations in this case is about

$$
C_{\text {recpt,pu }}=0.34
$$

This coefficient is almost the same as in the case with the uniform acoustic disturbances impinging at 68 degrees with the flat plate. This implies that the initiation of the instability wave occurs near the leading-edge region, and the acoustic disturbances in the downstream part, even though its amplitude is increasing, did not enhance the amplitude of the instability wave.

\section{E. Interaction of Acoustic Disturbances with a Cone}

We performed several simulations to understand the experimental observations ${ }^{14}$ (Fig. 1) on the generation of disturbances inside the boundary layer over an axisymmetric cone placed in an axisymmetric nozzle. The form of the disturbances in a cylindrical coordinate system can be written as

$$
q(x, r, \theta, t)=\tilde{q}(x, r) e^{i m \theta-i \omega t}
$$


where $\tilde{q}(x, r)$ is the amplitude variation. We first considered the interaction of two-dimensional $(m=0)$ uniform and nonuniform acoustic disturbances with the cone. Then we considered the interaction of three-dimensional $(m \neq$ 0 ) nonuniform acoustic disturbances with the cone. We performed the simulations for frequencies $f=10 \mathrm{kHz}$ and 50 $\mathrm{kHz}$ and for azimuthal wavenumbers $m=10,20$ and 30. The acoustic disturbances for the nonuniform cases were obtained from the wavy wall model described previously, Eqs. 9 and 10.

\section{Interaction of Uniform Two-Dimensional Acoustic Waves with the Cone}

For reference, we present the results obtained for the interaction of a two-dimensional $(m=0)$ plane acoustic wave with uniform amplitude and the cone. After the mean flow was computed, two-dimensional uniform acoustic disturbances were introduced at the outer boundary of the computational domain and time-accurate simulations were performed. The amplitude of these forced acoustic disturbances was assigned a small value of $\tilde{p}_{a c} / p_{\infty}=1 * 10^{-5}$ to ensure that the disturbances evolving in the boundary layer remained in the linear regime. Figure 17 shows the unsteady density fluctuations at a fixed time for the frequency of $10 \mathrm{kHz}$, and Fig. 18 shows the amplitude of the pressure fluctuations along the cone surface for the frequencies 10 and $50 \mathrm{kHz}$. As we observed from the N-Factor results ( Fig. 3(b) ), two-dimensional disturbances have very mild growth. The results show that the low-frequency disturbances, $f=10 \mathrm{kHz}$, decay in the axial direction and the high-frequency, $f=50 \mathrm{kHz}$, disturbances grow by about three times by the end of the cone.

\section{Interaction of Nonuniform Two-Dimensional Acoustic Waves with the Cone}

The results obtained from the simulation of the interaction of a radiated acoustic field originating from the nozzle wall and the cone are presented in Figs. 19-21. The results are shown for the frequency of $10 \mathrm{kHz}$ in these figures. Figure 19(a) shows the mass-flux fluctuations generated by the acoustic field interacting with the cone at a fixed time. The convective speed of the acoustic disturbances is 0.2 times the freestream velocity. The constant phase lines of the radiated noise are inclined at 68 degrees to the vertical axis. The amplitude of the radiated noise increases slowly along the axial direction similar to the effect observed in the experiment. It is seen that the acoustic field is reflected by the cone surface. Figure 19(b) depicts the maximum mass-flux fluctuations inside the boundary layer and the mass-flux fluctuations at the edge of the boundary layer. Note that even though the linear stability theory predicts that the disturbances should decay, the disturbances grow gradually in the axial direction. It is also observed that the maximum mass-flux fluctuations inside the boundary layer follow the fluctuations at the edge of the boundary layer. This is due to the local response of the boundary layer to the acoustic forcing at the boundarylayer edge. The amplitude inside the boundary layer is about 2 times the maximum amplitude at the edge of the boundary layer. We observed in the analysis of the response of the boundary layer to direct external acoustic forcing, Fig. 12(a), that the maximum amplitude inside the boundary layer is about 4 times larger than at the edge of the boundary layer at large incidence angles. We do not know at this point the reason for the observed lower amplitude ratio in the simulation, but the trend agrees with the analysis. In Figure 20, we plot the variation of the wavenumber of the pressure fluctuations along the wall. It is seen that the wavenumbers of the disturbances inside the boundary layer remain at a constant value equal to the wavenumber in the outer part of the boundary layer. This supports the conclusion that the disturbances we observed inside the boundary layer are the response of the external forcing at the boundary-layer edge. Figure 21 shows the amplitude variation in the normal direction at different axial locations of $x=0.5,3,6,10$, and 12 inches. Here $Y_{n}$ is the distance normal to the surface. Figure 21(a) displays the results inside and outside of the boundary layer, and Fig. 21(b) displays the results inside the boundary layer. Figure 21(a) shows the oscillations outside the boundary layer caused by the reflection of the incident wave by the cone. It is also seen that the amplitudes are about two times larger than that of the incoming wave. We also observe that the shape of the amplitude variation is similar to that obtained for the forced response of the boundary layer shown in Fig. 12(a) for the incident angle of 65 degrees.

Similarly, Figs. 22 and 23 show the simulation results for the frequency $f=50 \mathrm{kHz}$. Figure 22 depicts the maximum mass-flux fluctuations inside the boundary layer and the mass-flux fluctuations at the edge of the boundary layer. The maximum amplitude inside the boundary layer is smaller than the fluctuations at the edge of the boundary layer for this frequency. The amplitude variation inside the boundary layer follows the amplitude variation of the external forcing along the cone. Figures 23(a) and (b) show the amplitude variation in the normal direction at different axial locations of $x=0.5,3,6$, and 10 inches. Figure 23(a) shows the oscillations outside the boundary layer caused by the reflection of the incident wave by the cone. It is also seen that the amplitudes inside the boundary layer are about 0.8 times the amplitude of the disturbances outside the boundary layer. It is also seen that the shape of the amplitude variation is similar to that obtained for the forced response of the boundary layer shown in Fig. 13(a) for the incident angle of 65 degrees. 


\section{Interaction of Nonuniform Three-Dimensional Radiated Acoustic Waves with the Cone}

In this section, we present the simulation results for the interaction of three-dimensional disturbances with frequencies $f=10$ and $50 \mathrm{kHz}$ and azimuthal wavenumbers $m=10,20$, and 30 and the cone. We note from the linear stability computations that the frequency and the wavenumber of the most amplified wave are $f=50 \mathrm{kHz}$ and $m=30$. The maximum N-Factor based on the maximum mass-flux fluctuations obtained from the linear PSE computations are about 9.1 at $x=12$ inches. The neutral point for these parameters is located close to $x=2$ inches. Hence, the expected total amplification if instability waves have been generated near the neutral point is about 9000 up to $x=12$ inches. This factor is about 5 for the instability wave with $f=10 \mathrm{kHz}$ and $m=30$.

Figures 24-26 show the simulation results for the frequency $f=10 \mathrm{kHz}$. Figure 24(a) shows the mass-flux fluctuations generated by the acoustic field interacting with the cone at a fixed time. The figure shows the results for an azimuthal wavenumber of $m=30$, and the results for the azimuthal wavenumber of $m=10$ are almost the same. The convective speed of the acoustic disturbances is 0.2 times the freestream velocity. The constant phase lines of the radiated noise are inclined at 68 degrees to the vertical axis. The amplitude of the radiated noise increases slowly along the axial direction similar to the effect observed in the experiment. As we noticed earlier in Figs. 8(a-c), there exists a quiet core region at the center of the nozzle with increasing azimuthal number. We observe that up to $x \sim 8$ inches the radiated disturbances do not interact with the surface of the cone. Beyond that point, only the acoustic disturbances interact with the cone and reflect back into the outer part of the boundary layer. Figure 24(b) depicts the maximum mass-flux fluctuations inside the boundary layer and the mass-flux fluctuations at the edge of the boundary layer. The amplitudes inside and outside the boundary layer follow almost parallel to each other up to the end of the cone. The amplitudes inside the boundary layer are about 1.25 times larger than the amplitude outside the boundary layer. We also included the linear amplification curve obtained from the PSE computations. The growth of the disturbances obtained from the simulations does not follow the curve obtained from the PSE until further downstream.

Figure 25 shows the variation of the wavenumber of the pressure fluctuations along the surface of the cone. We also include for comparison the wavenumber variation for the linear stability wave. This shows that the disturbances inside the boundary layer are not the instability modes. Figures 26(a) and (b) show the amplitude variation in the normal direction at different axial locations of $x=7,8,10$, and 13 inches for the azimuthal wavenumbers $m=10$ and 30, respectively. It is seen that the amplitudes are about 1.25 times the amplitude of the disturbances outside the boundary layer. We also plot the eigenfunctions obtained from the PSE computations for the azimuthal wavenumber of $m=30$ in Fig. 26(b). It is noted that the shape of the amplitude variation inside the boundary layer is similar to the forced response of the boundary layer shown in Fig. 13(b) for the incident angle of 65 degrees. This implies that the disturbances generated inside the boundary layer are not the instability modes but rather the forced disturbances from the external acoustic disturbances.

Similarly, Figures 27 and 28 show the simulation results for the frequency $f=50 \mathrm{kHz}$. Figure 27 depicts the maximum mass-flux fluctuations inside the boundary layer and the mass-flux fluctuations at the edge of the boundary layer for the azimuthal wavenumber of $m=30$. The maximum amplitude inside the boundary layer is much smaller than the fluctuations at the edge of the boundary layer for this frequency. The amplitude variation inside the boundary layer approximately follows the amplitude variation of the external forcing along the cone. We also included the linear amplification curve obtained from the PSE computations. As we mentioned previously, according to linear theory the mass-flux fluctuations should have grown by about 9000 or four orders by the end of the cone. This large growth is not observed in the simulation results. Figures 28 (a) and (b) show the amplitude variation in the wall-normal direction at different axial locations of $x=7,8,10$, and 13 inches for the azimuthal wavenumbers $m=10$ and 30. The figures show that the fluctuations inside the boundary layers are about 5 times smaller than in the outer part of the boundary layer.

One of the important conclusions from these simulations at two frequencies and different azimuthal wavenumbers is that instability waves are not generated by these three-dimensional acoustic disturbances impinging at highly oblique angles of 68 degrees or the acoustic sources propagating at a speed of 0.2 times the freestream velocity. The other conclusion is that disturbances generated inside the boundary layers are the forced response of the boundary layers to the external acoustic forcing.

In all the previous simulation results, we considered acoustic disturbances propagating at a low convecting speed of 0.2 times the freestream velocity. We performed those simulations based on the experimental observation ${ }^{14}$ that in the transitional zone of the axisymmetric nozzle in SLDT the noise sources are propagating at this speed. But experimental observations ${ }^{22-24}$ and direct numerical simulations ${ }^{24}$ show that noise sources in two-dimensional nozzle wall boundary layers and in flat-plate turbulent boundary layers propagate close to $0.4 \sim 0.5$ times the freestream velocity at supersonic Mach numbers. Additionally, measurements of source velocity $\sim 0.2$ to 0.5 times the freestream velocity were done in the 2D nozzle of SLDT at Mach 3.5. ${ }^{15}$ Hence we performed simulations with the 
noise sources propagating at a speed of 0.6 times the freestream velocity. We considered both frequencies $f=10$ and $50 \mathrm{kHz}$ and did not find any large disturbances inside the boundary layer at the lower frequency $f=10 \mathrm{kHz}$. Simulations performed at the azimuthal wavenumber of $m=30$ also did not reveal any large perturbations inside the boundary layer. Hence we present the results for a frequency of $f=50 \mathrm{kHz}$ and an azimuthal wavenumber of $m=20$.

Figures 29-31 show the simulation results for this case. Figure 29(a) shows the mass-flux fluctuations generated by the acoustic field interacting with the cone at a fixed time. The constant phase lines of the radiated noise are inclined at 45 degrees to the vertical axis. The amplitude of the radiated noise increases slowly along the axial direction similar to the effect observed in the experiment. As we noticed in the previous case, there exists a quiet core region at the center of the nozzle. We observe that up to $x \sim 8$ inches, the radiated disturbances do not interact with the surface of the cone. Figure 29(b) depicts the maximum mass-flux fluctuations inside the boundary layer and the mass-flux fluctuations at the edge of the boundary layer. The amplitudes inside and outside the boundary layer follow almost parallel to each other up to the end of the cone. The amplitudes inside the boundary layer are about 2.5 times larger than in the boundary-layer edge. We also included the linear amplification curve obtained from the PSE computations. The growth of the disturbances obtained from the simulations follows the curve obtained from the PSE from $x=11$ to 14 inches.

Figure 30 shows the variation of the wavenumber of the pressure fluctuations along the surface of the cone. We also include for comparison the wavenumber variation for the linear stability wave. It is seen that, the wavenumbers obtained from the simulation and the linear stability theory differs along the cone. This also shows that the disturbances inside the boundary layer are not the instability modes. Figures 31(a) and (b) show the amplitude variation in the normal direction at different axial locations of $x=7,8,10,11,12$ and 14 inches for the azimuthal wavenumbers $m=20$. It is seen that the amplitudes are about 2.5 times the amplitude of the disturbances outside the boundary layer. We also plot the eigenfunction distributions obtained from the PSE computations at $x=12$ and 14 inches in Fig. 31(b). It is also seen that the shape of the amplitude variation is similar to that for the forced response of the boundary layer shown in Fig. 13(b) for the incidence angle of 65 degrees. However, near the wall, the eigenfunction from the PSE agrees in shape with that from the simulation.

\section{Discussions and Conclusions}

Our main objective in this study was to understand or explain the findings from the detailed experimental data gathered in the SLDT at $\mathrm{M}=3.5$ over an axisymmetric 7-deg half-angle cone. ${ }^{14}$ Before we performed the experiment we expected from the linear stability computations that high-frequency disturbances with large azimuthal wavenumbers would exponentially grow and dominate the transition process. In contrary, the experimental data revealed that the low-frequency disturbances have larger amplitudes than the high-frequency disturbances, and further, the high-frequency disturbances had limited growth on the order of $\sim 10$, compared to the expected exponential growth on the order of $\sim 10^{4}$. Another interesting finding from the experiment was that the amplitude of the disturbances outside and inside the boundary layer grew almost in parallel along the cone and the ratios of the amplitudes between the inside and outside was approximately 5 to 7 .

Since the linear disturbances inside a boundary layer consist of eigen solutions and the forced solutions of the linearized Navier-Stokes equations, we first computed the forced response of supersonic boundary layers over a flat plate and a cone. The results for the flow over a flat plate showed that the maximum amplitude of the disturbances inside the boundary layer are about 0.8 times the maximum amplitude outside of the boundary layer. The computations also showed that the response increases with the acoustic incident angle.

For the cone, the response of the forcing is stronger in the cone boundary layer than in the flat-plate boundary layer. The computations at low frequency for two-dimensional disturbances $(m=0)$ showed that the ratio between the maximum amplitudes inside and outside the boundary layer is about 5.2 for an incident angle of 30 degrees. This ratio is about 4 for the incident angle of 65 degrees. The results for the higher azimuthal wavenumber of $m=30$ showed that the responses decrease compared to the two-dimensional case. The ratio between the maximum amplitudes inside and outside the boundary layer is about 1.4 for the incident angle of 65 degrees. This is about 4 times smaller than at the azimuthal wavenumber of $m=0$. The maximum amplitudes of the eigenfunctions obtained from the forcing and the linear stability theory occurred at the same $\eta$ location, but the shapes were different in the lower part of the boundary layer. The results at higher frequency for two-dimensional disturbances showed that the ratio between the maximum amplitudes inside and outside the boundary layer is about 2.5 for the incident angle of 30 degrees. In the larger azimuthal wavenumber case for $m=30$, the amplitude ratio between the maximum amplitudes inside and outside the boundary layer was about 1.0 for the incident angle of 30 degrees.

We investigated the generation and evolution of disturbances generated by uniform and nonuniform acoustic disturbances in supersonic flows over a flat plate and a sharp cone. The nonuniform acoustic field radiated from the 
nozzle wall was modeled by placing a convecting wavy wall at the surface of the nozzle walls. The solution is obtained using the Fourier transform method. The acoustic field was superimposed at the outer boundary of the computational domain and time-accurate simulations were performed to investigate the interaction of this field with the flat plate and the sharp cone at zero angle of attack.

The simulations for the flat plate in a two-dimensional nozzle show that acoustic disturbances always generate instability waves near the leading edge of the plate irrespective of the incident angle. The receptivity coefficient is about 0.5 based on the wall pressure fluctuations near the neutral point when the acoustic disturbances impinged at zero incident angle. However, the results show that the receptivity coefficients become about 20 times smaller when the acoustic disturbances are incident at highly oblique angles. The simulation with nonuniform acoustic forcing shows that increasing the freestream amplitude along the flat plate does not enhance the receptivity process or the receptivity coefficient. Hence, in high-speed tunnels where the radiated noise impinges the plates at high incident angles, the initial amplitudes of the disturbances will be much smaller than those generated by a plane wave at zero incidence angles.

The simulations for the cone in an axisymmetric nozzle with a two-dimensional plane acoustic wave show that the low-frequency disturbances decay in the axial direction and the high-frequency disturbances grow mildly by a factor of two within the cone length. We performed several simulations with nonuniform acoustic forcing radiated by the nozzle wall. This forcing mimicked the experimentally observed forcing at the edge of the boundary layer of the cone. The simulations with two- and three-dimensional nonuniform disturbances at low and high frequencies with low and high azimuthal wavenumbers did not show any generation of instability waves by these external disturbances. The results in general showed that the maximum mass-flux fluctuations inside the boundary layer follow the fluctuations at the edge of the boundary layer. This is due to the local response of the boundary layer to the acoustic forcing at the boundary-layer edge. The amplitude inside the boundary layer is about 2 times the amplitude at the edge of the boundary layer. It appears from this study that the observed disturbances inside the boundary layer are the response of the boundary layer to the external forcing at the boundary-layer edge.

There are questions still lingering about the transition process over the cone in an axisymmetric wind tunnel environment. The experiment could not go far enough along the cone to detect the transition onset and the transition end regions due to the limited travel range of the traverse at this stagnation pressure. As we noted in the experiment, the amplitude of the low-frequency disturbances at the end of the measurement station is about 2 times larger than the high-frequency disturbances. Further, the amplitudes of the disturbances inside and outside the boundary layer continue to grow downstream. Will the high-frequency disturbances eventually take off and cause the transition? There is evidence in the experimental ${ }^{14}$ spectra that the high-frequency range shows faster growth at the end of the measurement region than in the low-frequency region. Another important question is about the content of the azimuthal wavenumbers of the most dominant disturbances inside the boundary layer. In the present experiment, only a single hot-wire was used to gather the perturbations inside the boundary layer. It was not possible to collect simultaneous data in the azimuthal direction to decompose the data into different azimuthal modes. In future experiments, techniques must be used to infer this information.

\section{References}

1. Mack, L. M., "Boundary Layer Stability Theory”, JPL 900-277 Rev. A, Nov. 1969, Jet Propulsion Lab., Pasadena, Calif.

2. Mack, L. M., "On the Application of Linear Stability Theory and the Problem of Supersonic Boundarylayer Transition," AIAA Journal., 13, No. 3, 1975.

3. Gaponov, S. A., "Interaction between a Supersonic Boundary Layer and Acoustic Disturbances," Fluid Dynamics, 6, 1977.

4. Gaponov, S. A., "Excitation of Instability Waves in the Supersonic Boundary Layer by Sound," IUTAM Symposium Potsdam, Springer-Verlag, 1993.

5. Gaponov, S. A. and Smorodsky, B. V., "Supersonic Boundary Layer Receptivity to Streamwise Acoustic Field," IUTAM Symposium,, Spinger-Verlag, 1999.

6. Fedorov, A. V., and Khokhlov, A. P., " Excitation of Unstable Modes in a Supersonic Boundary Layer by Acoustic Waves," Fluid Dynamics, No. 9, pp. 456-467, 1991. 
7. Fedorov, A. V., and Khokhlov, A. P., "Sensitivity of a Supersonic Boundary Layer to Acoustic Disturbances," Fluid Dynamics, No. 27, pp. 29-34, 1992.

8. Sakaue, S., Asia, M., and Nishioka, M., "On the Receptivity Process of Supersonic Laminar Boundary Layer", Laminar Turbulent Transition, Heidelberg, Springer-Verlag, 2000.

9. Fedorov, A. V., "Receptivity of High Speed Boundary Layer to Acoustic Disturbances," J. Fluid Mechanics, Vol.491, pp. 101-129, 2003.

10. P. Balakumar, "Transition in a Supersonic Boundary layer Due to Roughness and Acoustic Disturbances," AIAA Paper 2003-3589, 2003.

11. P. Balakumar, "Receptivity of a Supersonic Boundary Layer to Acoustic Disturbances," AIAA Journal, Vol. 47, No. 5, May 2009, pp. 1034-1041.

12. P. Balakumar, "Stability of a Supersonic Boundary layer Over Blunt Wedges," AIAA Paper 2006-0137, 2006.

13. Chen, F. J., Malik, M. R., Beckwith, I. E., "Comparison of Boundary Layer transition on a Cone and Flat Plate at Mach 3.5," AIAA-88-0411, 1988.

14. King, R. A., Chou, A., Balakumar, P., Owens, L. R., and Kegerise, M. A., "Measurements in a Transitioning Cone Boundary Layer at Freestream Mach 3.5”, AIAA Paper 2016-0050, 2016, San Diego, CA.

15. Beckwith I. E., Creel, T. R. Jr., Chen, F. -J., and Kendall, J. M., "Free-Stream Noise and Transition Measurements on a Cone in a Mach 3.5 Pilot Low-Disturbance Tunnel," NASA TP-2180, 1983, (also AIAA Paper 83-0042).

16. Shu, Chi-Wang, "Essentially Non-Oscillatory and Weighted Essentially Non-Oscillatory Schemes for Hyperbolic Conservation Laws," NASA/CR-97-206253 and ICASE Report N0. 97-6

17. Atkins, H. L., "High-Order ENO Methods for the Unsteady Compressible Navier-Stokes Equations," AIAA Paper 91-1557, 1991.

18. Balakumar, P., Zhao, H., and Atkins, H., "Stability of Hypersonic Boundary-layers Over a Compression Corner," AIAA Paper 2002-2848, 2002.

19. Balakumar, P., and Kegerise, M. K., "Receptivity of Hypersonic Boundary Layers to Acoustic and Vortical Disturbances," AIAA 2011-0371, 49 ${ }^{\text {th }}$ AIAA Aerosciences Meeting, Orlando, Florida, January 4-7, 2011.

20. Balakumar, P., Malik, M. R., "Discrete Modes and Continuous Spectra in Supersonic Boundary Layers," J. Fluid Mechanics, Vol. 239, 1992, pp. 631-656.

21. Tumin, A. ,'Three-Dimensional Spatial Normal Modes in Compressible Boundary Layers", J. Fluid Mechanics, Vol. 586, pp. 295-322, 2007.

22. Laufer, J., "Aerodynamic Noise in Supersonic Wind Tunnels," Journal of the Aerospace Sciences, Vol. 28, September 1961, pp. 685-692.

23. Laufer, J., "Sound Radiation from a Turbulent Boundary Layer," In Proceedings of the Marseille Conference on Turbulence. CNRS Report 108, 1962.

24. Laufer, J. "Some Statistical Properties of the Pressure Field Radiated by a Turbulent Boundary Layer," Phys. Fluids 7 (8), 1964, 1191-1197.

25. Duan, L., Choudhari, M. M., Wu, M., "Numerical Study of Acoustic Radiation due to a Supersonic Turbulent Boundary Layer," J. Fluid Mechanics, Vol. 746, 2014, pp. 165-192. 
(a) $f=10 \mathrm{kHz}$

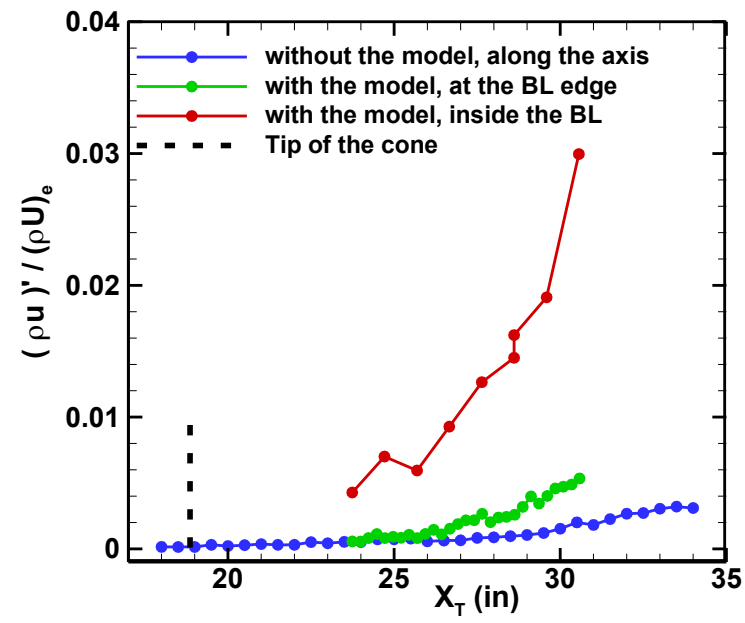

(b) $f=50 \mathrm{kHz}$

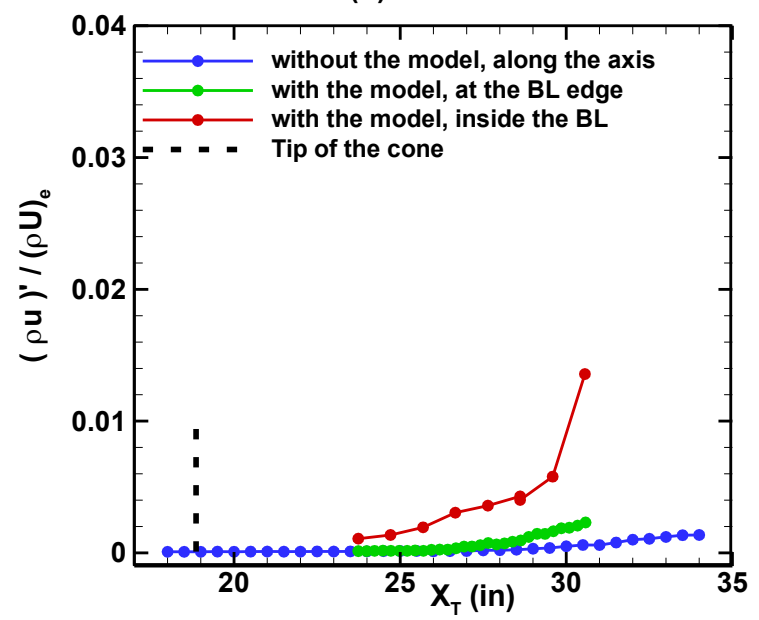

Figure 1. Measured mass-flux fluctuations amplitude for different frequencies on linear-scale. (a) $10 \mathrm{kHz}$, and (b) $50 \mathrm{kHz}$.

(a) $f=10 \mathrm{kHz}$

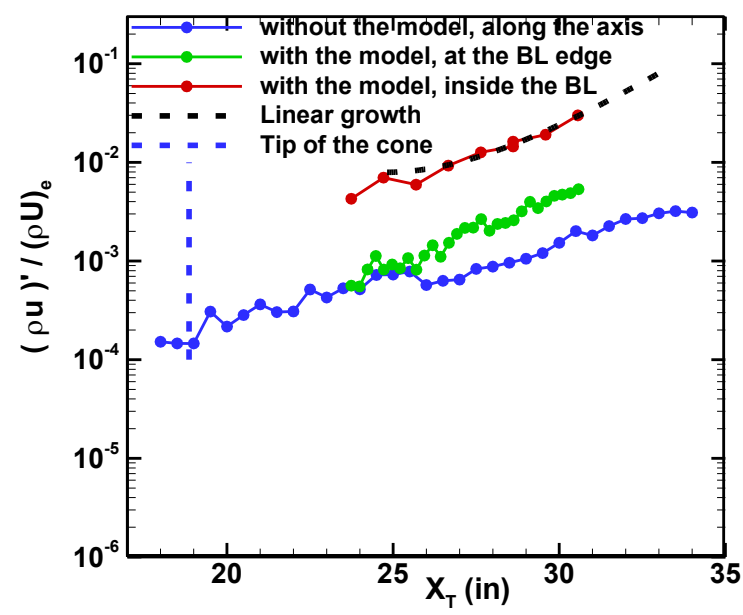

(b) $f=50 \mathrm{kHz}$

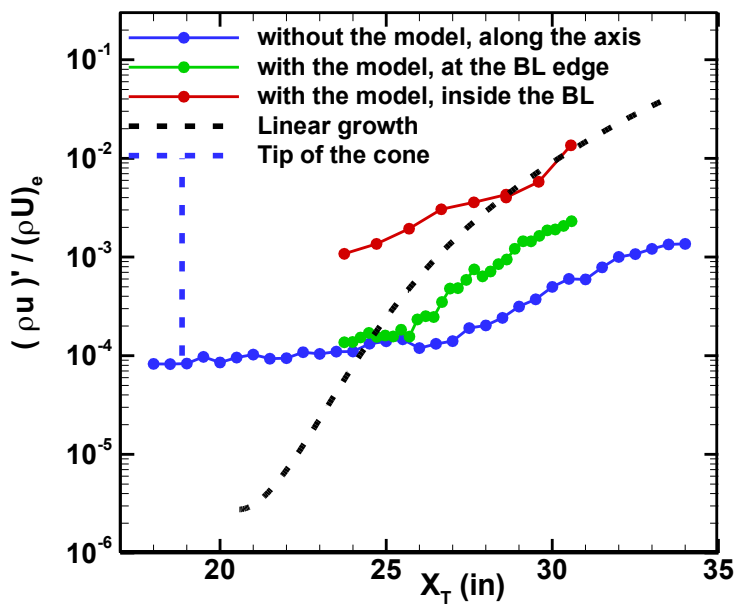

Figure 2. Measured mass-flux fluctuation amplitude and the growth predicted from linear theory for different frequencies on $\log$-scale. (a) $10 \mathrm{kHz}$, and (b) $50 \mathrm{kHz}$. 

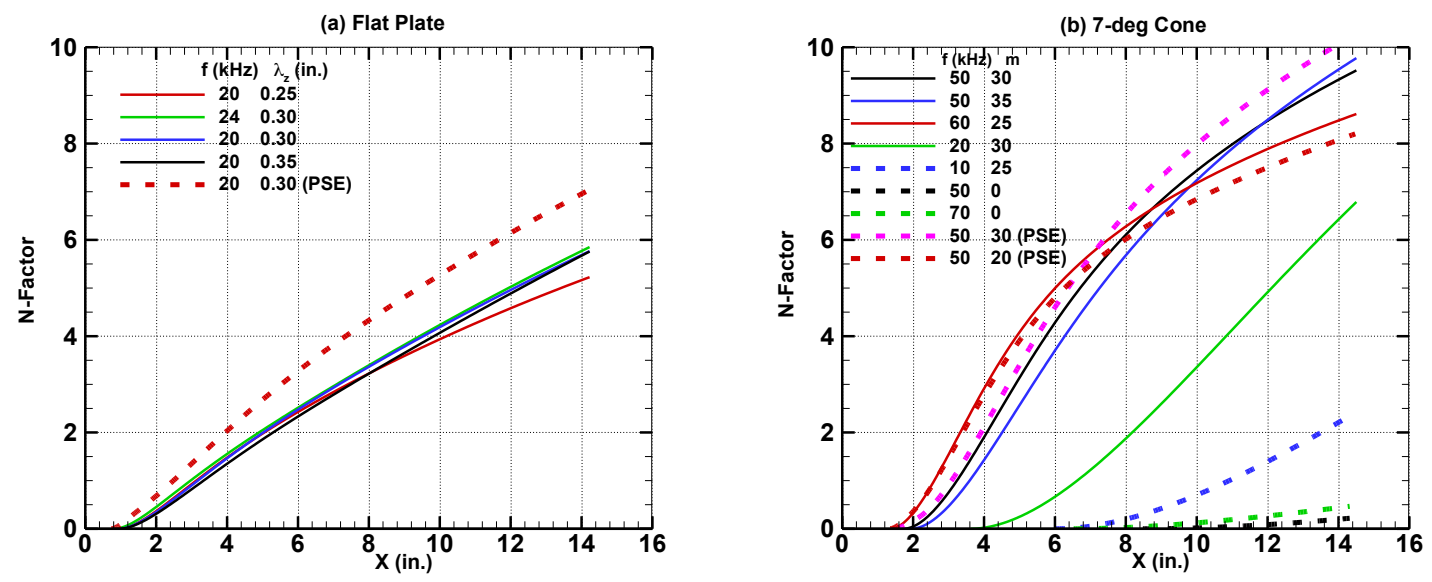

Figure 3. N-Factor curves obtained from linear stability calculations for (a) flat plate, and (b) cone.
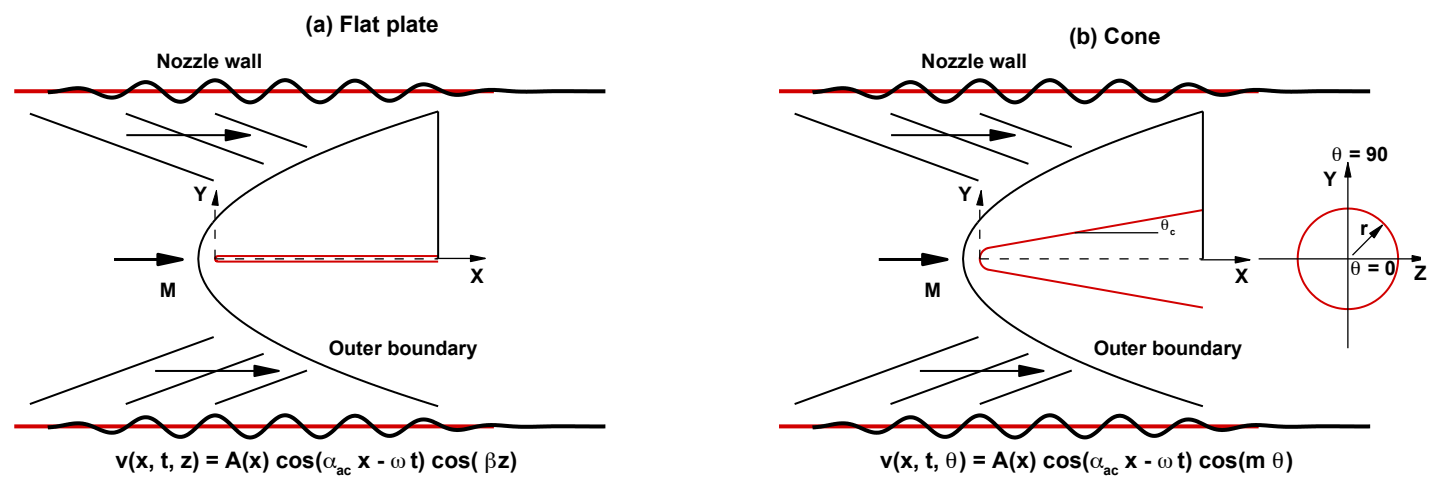

Figure 4. Schematic diagram of the computational setup (a) for the flat plate, and (b) for the cone.

(a)

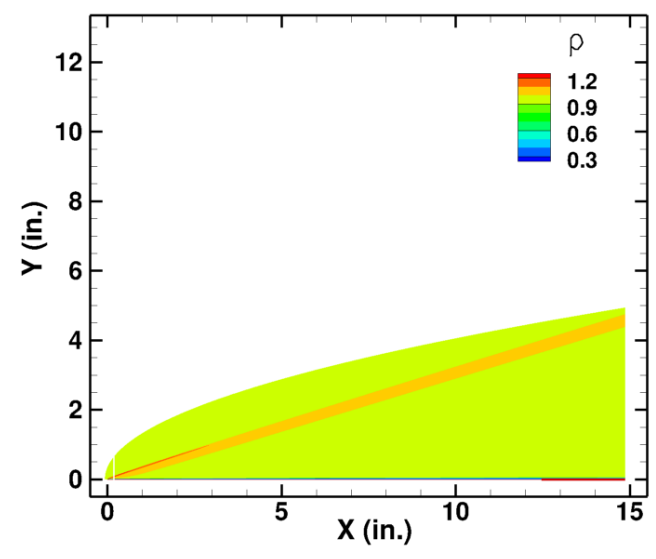

(b)

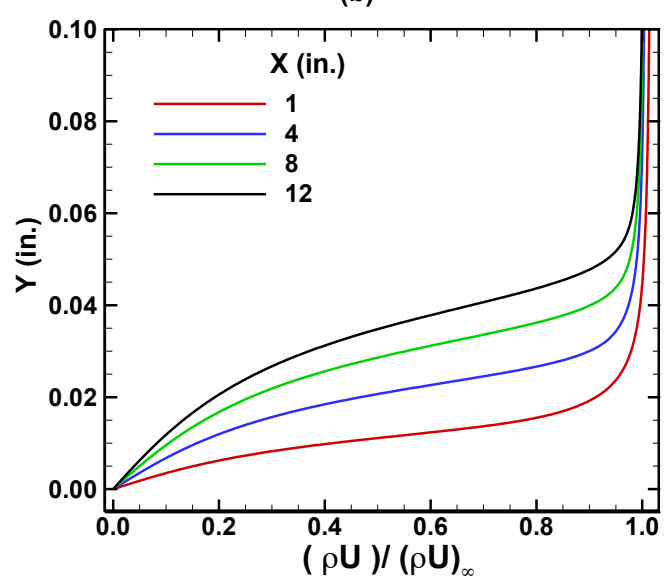

Figure 5. Mean flow field over the flat plate. (a) Density contours, and (b) boundary layer profiles for the mass flux. 
(a)

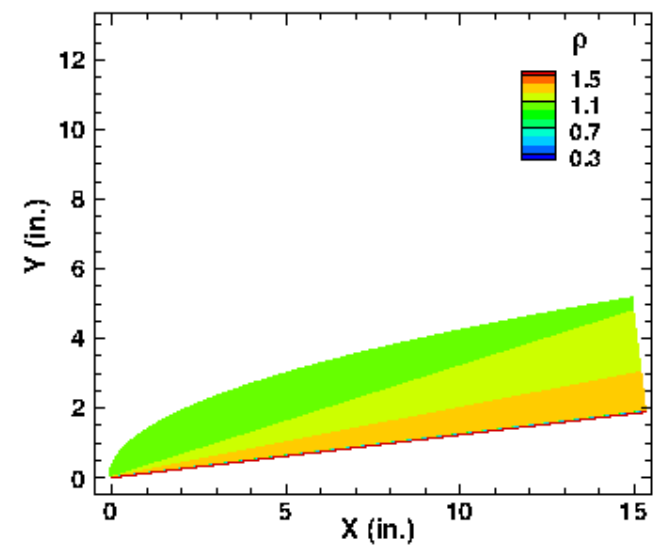

(b)

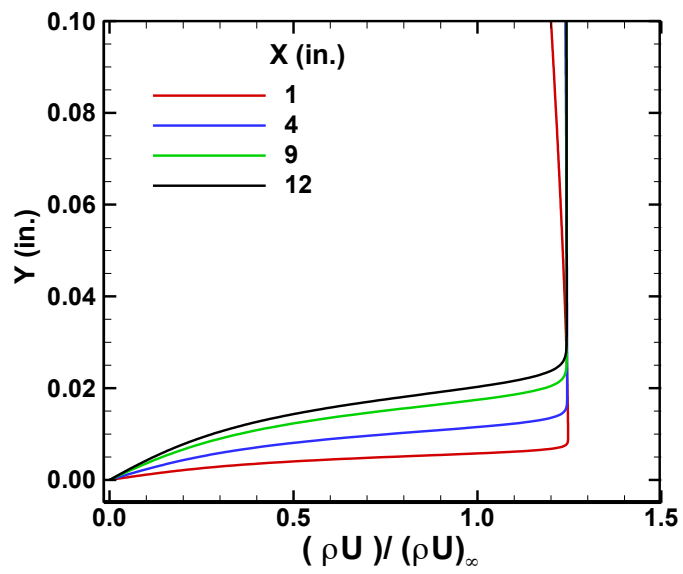

Figure 6. Mean flow field over the cone. (a) Density contours, and (b) boundary layer profiles for the mass flux.

(a) Flat plate, $\mathrm{f}=20 \mathrm{kHz}, \lambda_{\mathrm{z}}=0.3 \mathrm{in}$.

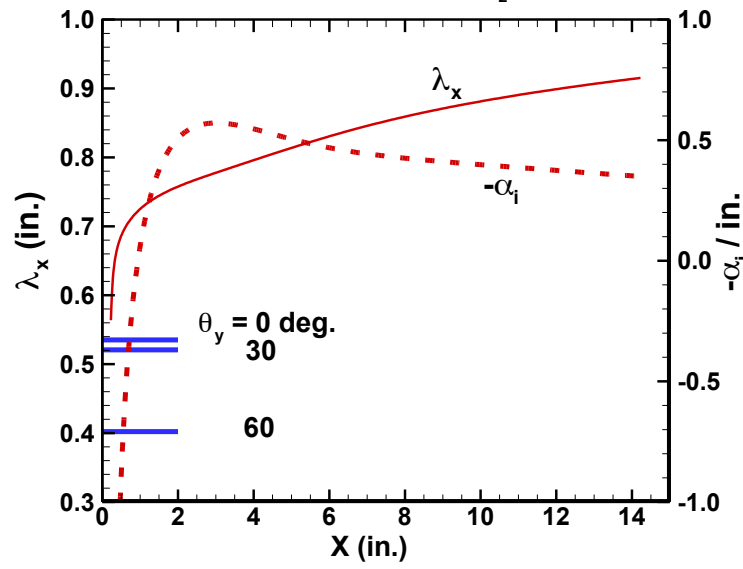

(b) Cone, f= $50 \mathrm{kHz}, \mathrm{m}=\mathbf{3 0}$.

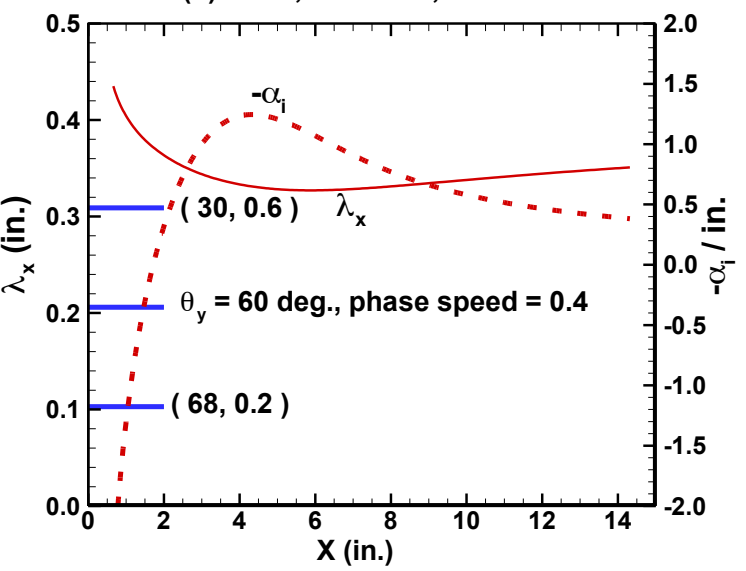

(c) Cone, $\mathrm{f}=10 \mathrm{kHz}, \mathrm{m}=\mathbf{3 0}$.

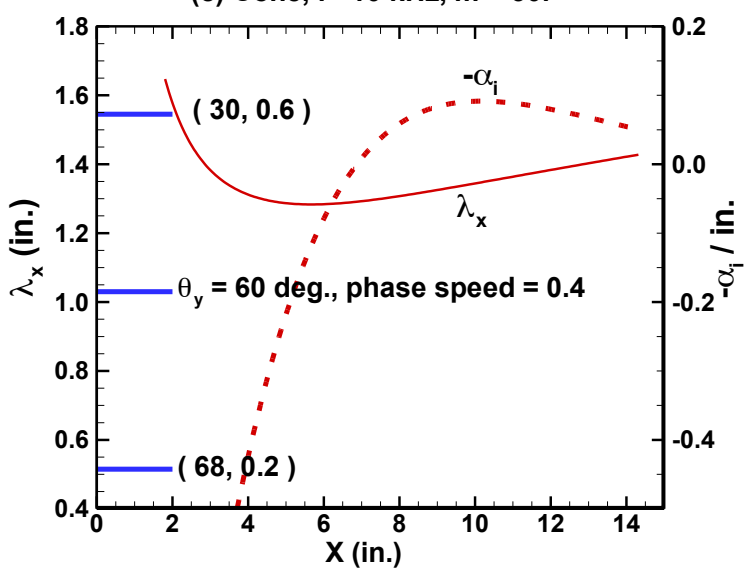

Figure 7. Variation of the axial wavelengths and the growth rates for (a) the flat plate $f=20 \mathrm{kHz}, \lambda_{z}=0.30$ in., and for the cone (b) $f=50 \mathrm{kHz}, m=30$, and (c) $f=10 \mathrm{kHz}, m=30$. 

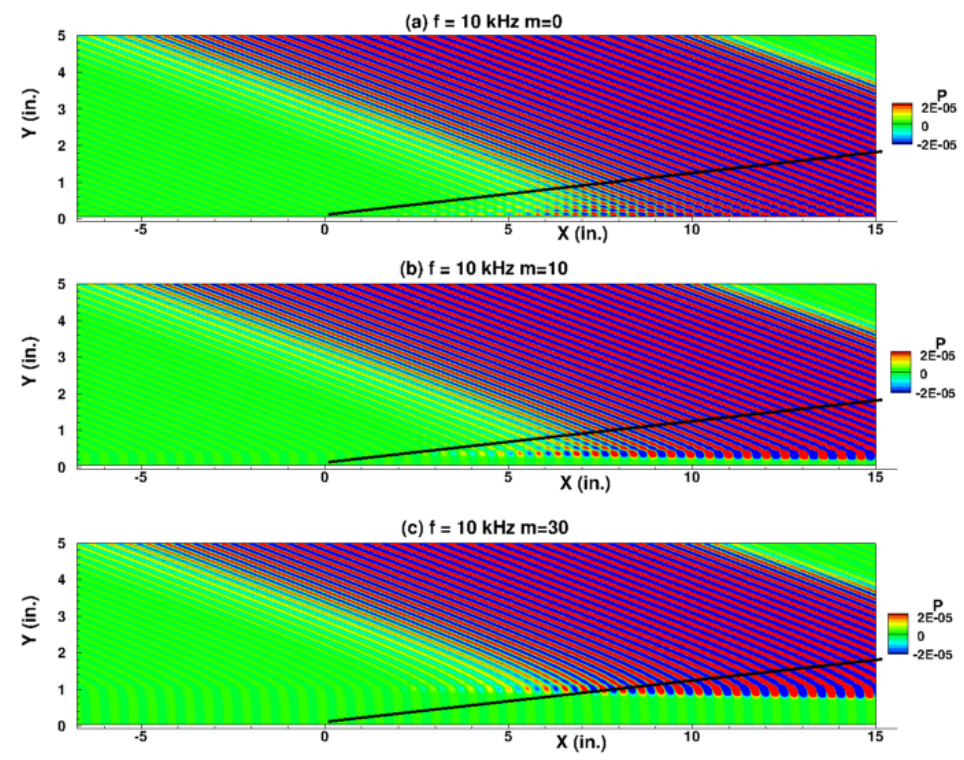

Figure 8. Radiated pressure field for $f=10 \mathrm{kHz}$ from a convecting wavy wall placed on an axisymmetric nozzle wall (a) $m=0$, (b) $m=10$, and (c) $m=30$.

(a) $\mathrm{f}=10 \mathrm{kHz}, \mathrm{m}=\mathbf{3 0}$

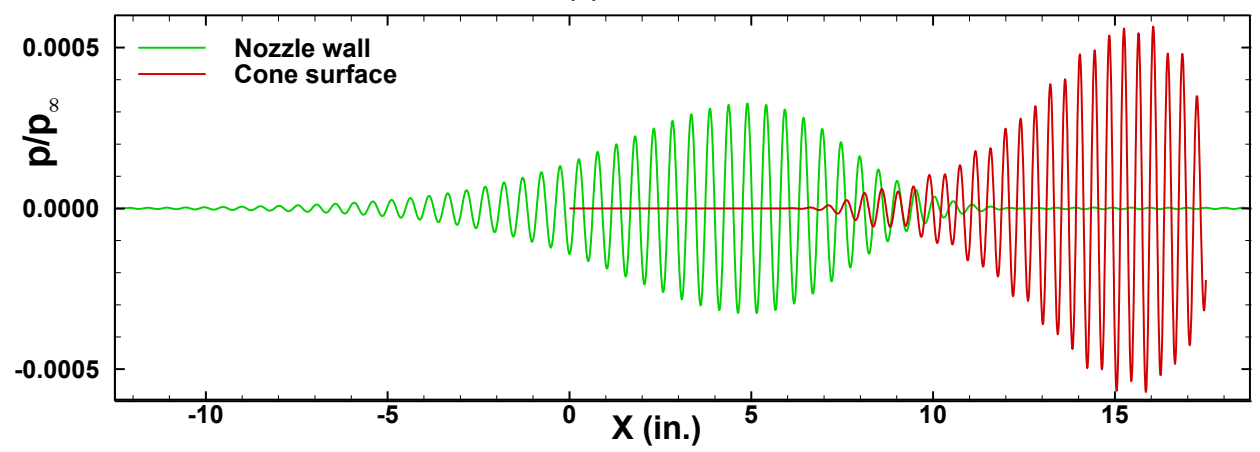

(b) $f=10 \mathrm{kHz}$

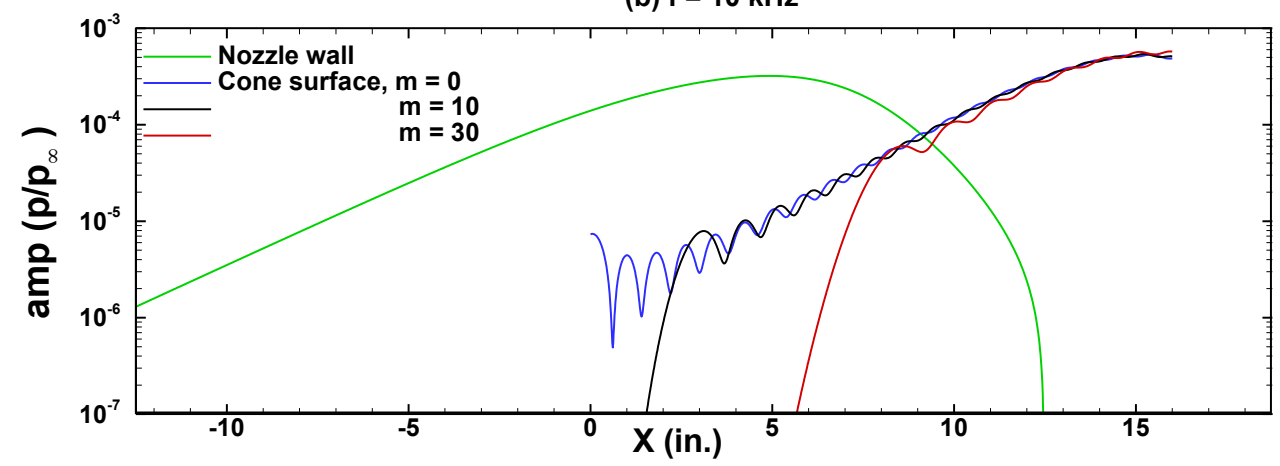

Figure 9. Pressure along the nozzle wall and the pressure field generated by the source along the cone surface for $f=10 \mathrm{kHz}$ (a) instantaneous pressure field, and (b) amplitude. 


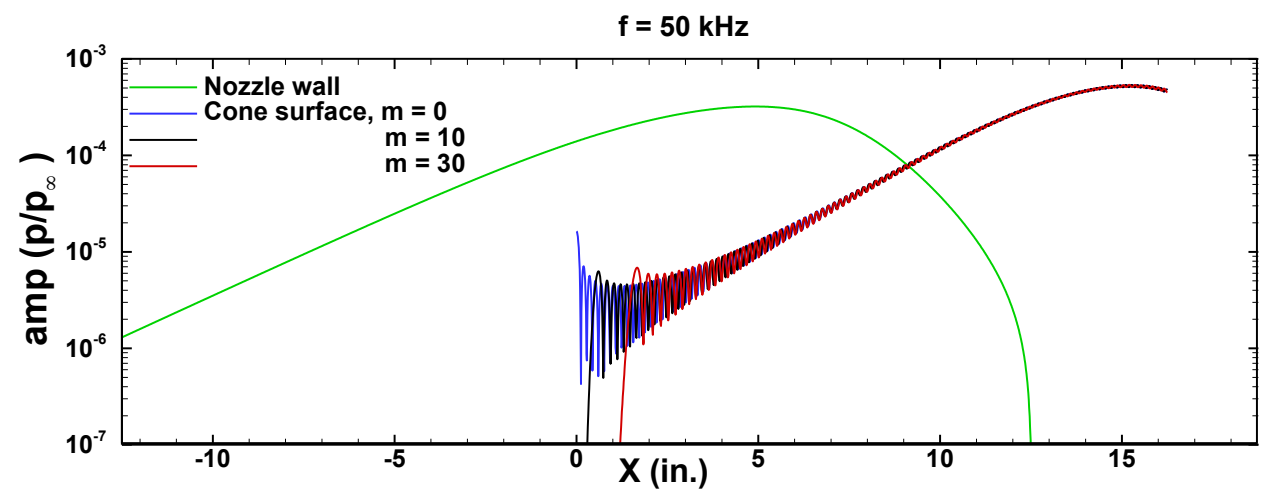

Figure 10. Pressure amplitude along the nozzle wall and the pressure field generated by the source along the cone surface. $f=50 \mathrm{kHz}$

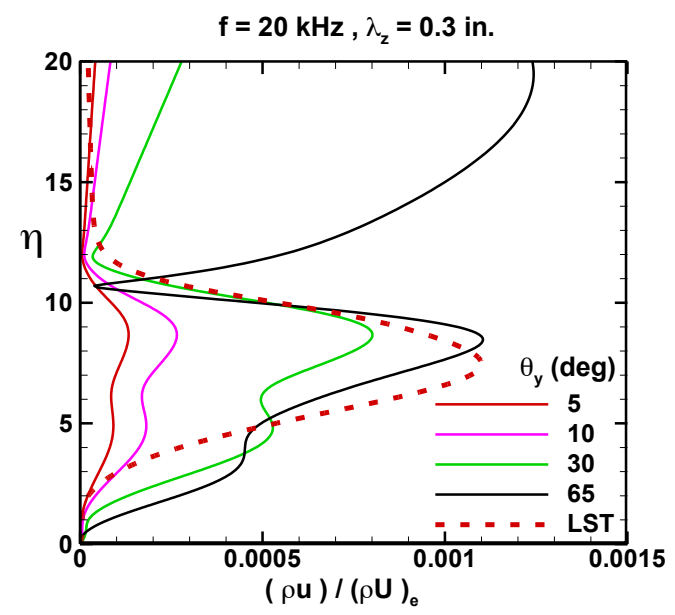

Figure 11. Amplitude of the mass-flux perturbations induced by the forced acoustic field at different incident angles for the flat plate boundary layer. $f=20 \mathrm{kHz}, R=2270, \lambda_{z}=0.30$ inches.

(a) $f=10 \mathrm{kHz}, \mathrm{m}=0$

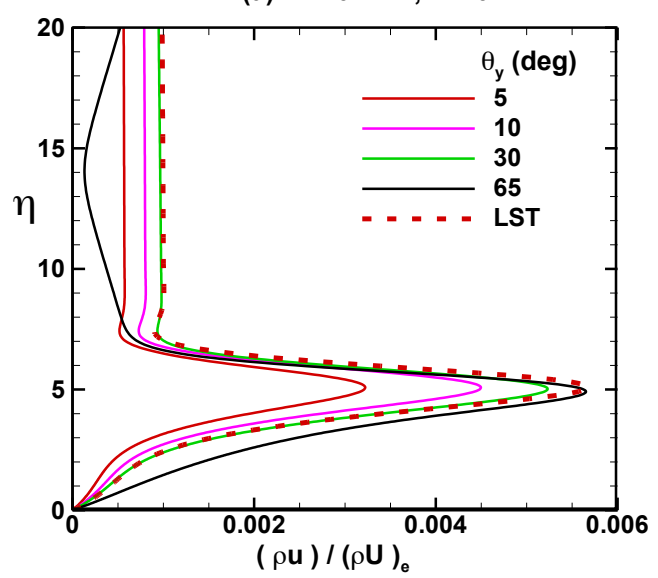

(b) $f=10 \mathrm{kHz}, \mathrm{m}=30$

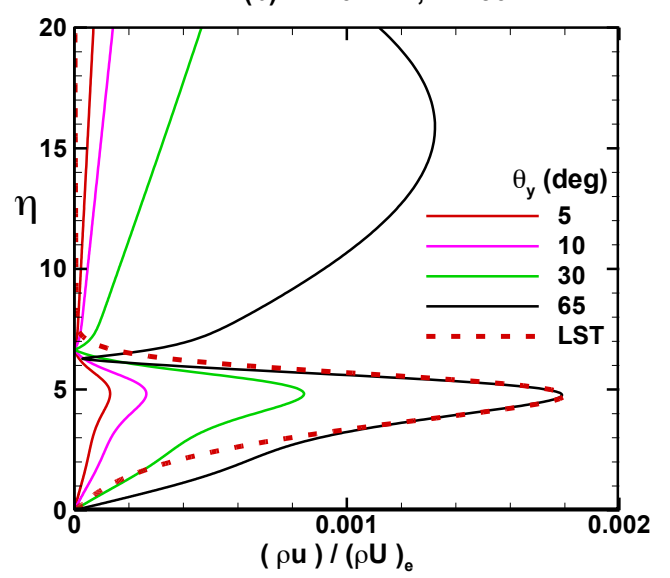

Figure 12. Amplitude of the mass-flux perturbations induced by the forced acoustic field at different incident angles for the cone boundary layer. $f=10 \mathrm{kHz}, R=2425$ (a) $m=0$, and (b) $m=30$. 
(a) $f=50 \mathrm{kHz}, \mathrm{m}=0$

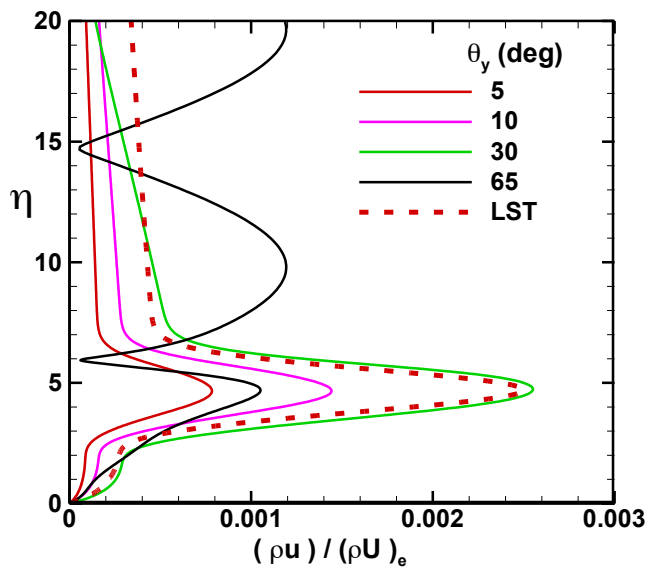

(b) $f=50 \mathrm{kHz}, \mathrm{m}=\mathbf{3 0}$

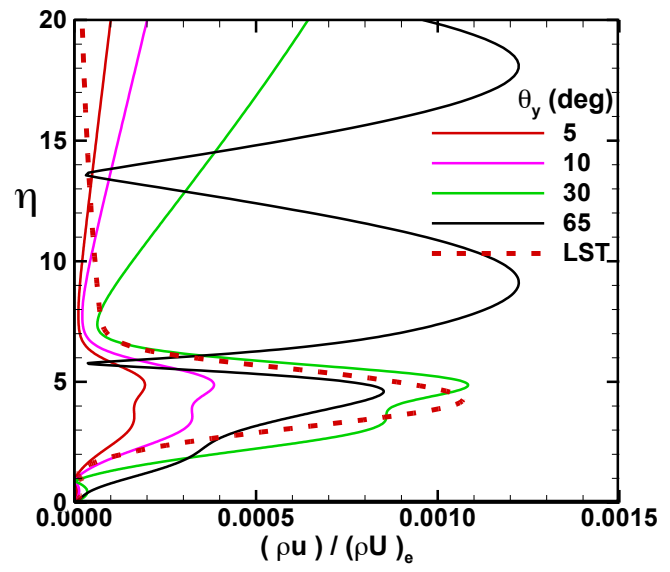

Figure 13. Amplitude of the mass-flux perturbations induced by the forced acoustic field at different incident angles for the cone boundary layer. $f=50 \mathrm{kHz}, R=2425$, (a) $m=0$, and (b) $m=30$.

(a) $f=20 \mathrm{kHz}, \lambda_{z}=0.3$ in.

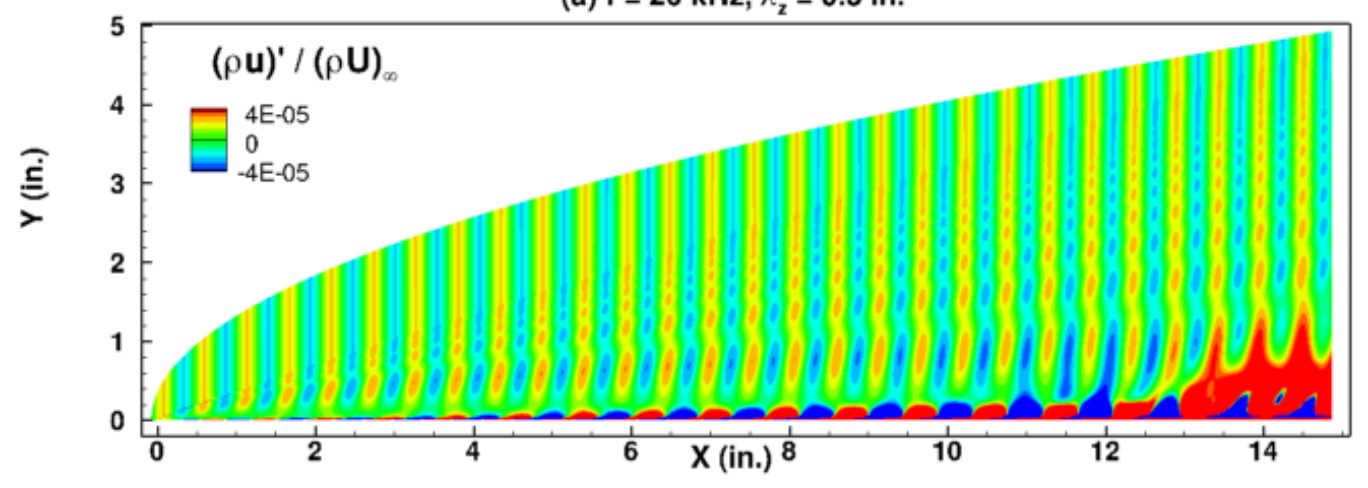

(b)

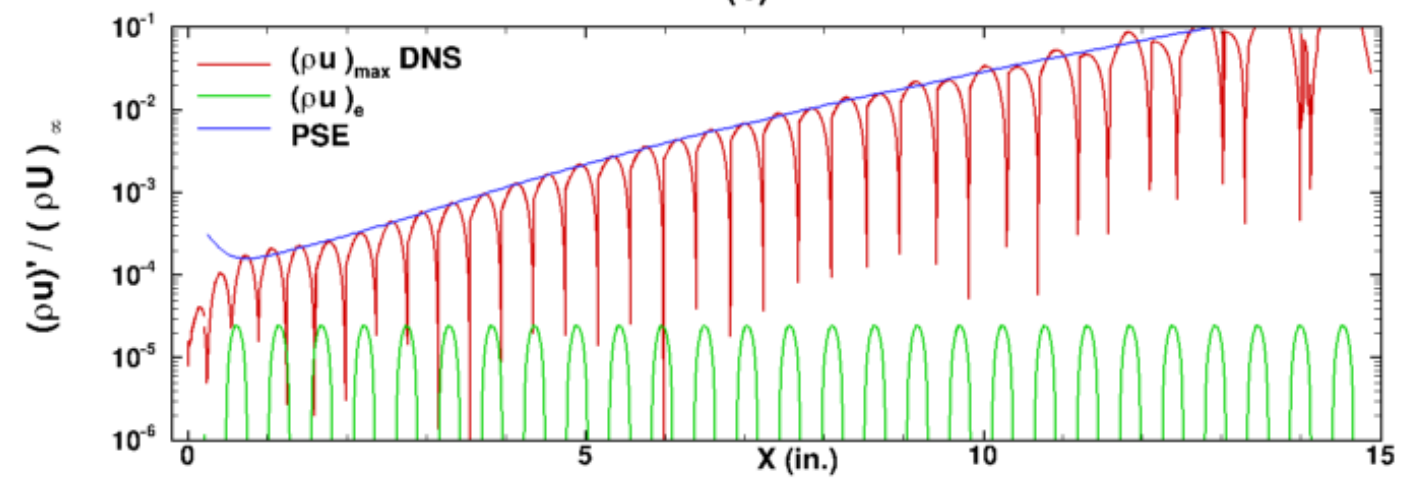

Figure 14. Fluctuations generated by the interaction of a uniform three-dimensional plane acoustic wave with a flat plate. $f=20 \mathrm{kHz}, \lambda_{z}=0.3 \mathrm{in}, \theta_{y}=0.0 \mathrm{deg}$. (a) mass-flux contours at a fixed time, and (b) maximum mass-flux fluctuations inside and outside the boundary layer. 
(a) $f=20 \mathrm{kHz}, \lambda_{z}=0.3$ in.

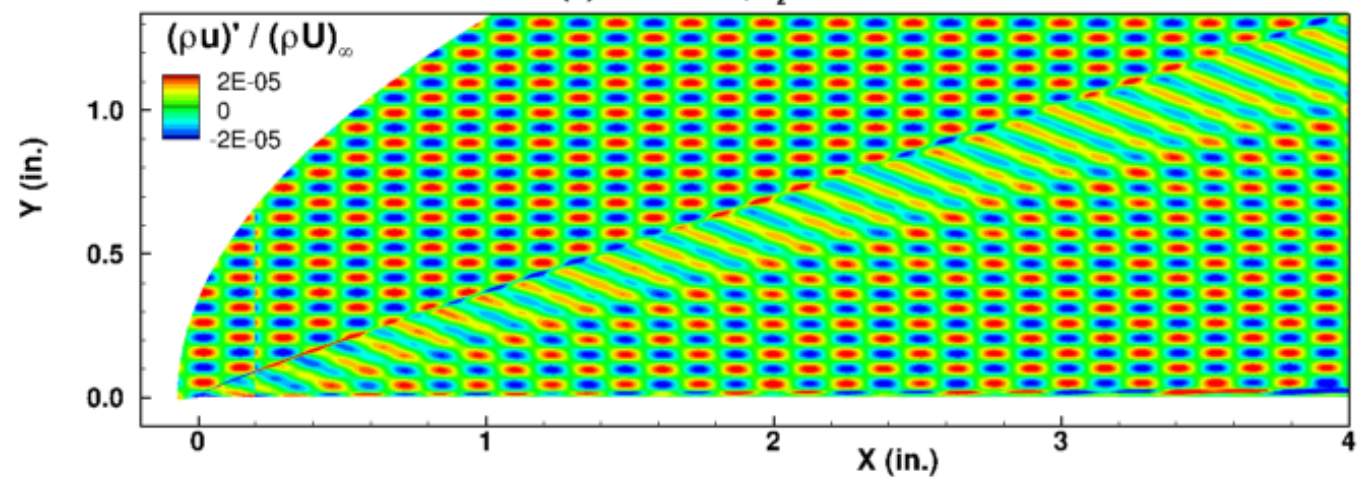

(b)

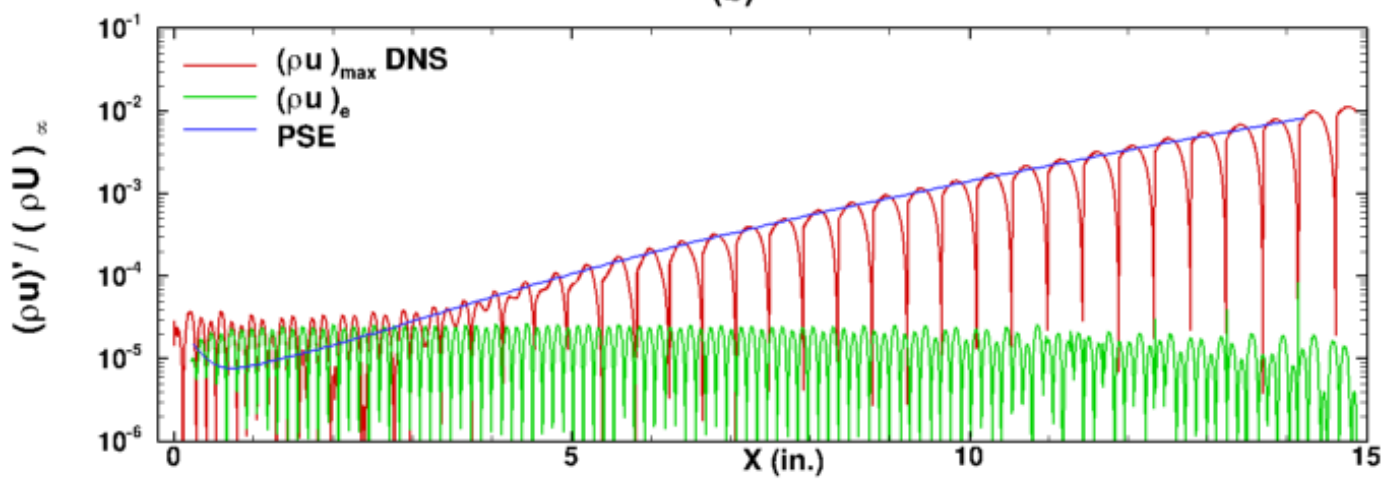

Figure 15. Fluctuations generated by the interaction of a uniform three-dimensional plane acoustic wave incident at 68 degrees with a flat plate. $f=20 \mathrm{kHz}, \lambda_{z}=0.3 \mathrm{in}, \theta_{y}=68.0 \mathrm{deg}$. (a) mass-flux contours at a fixed time, and (b) maximum mass-flux fluctuations inside and outside the boundary layer. 
(a) $f=20 \mathrm{kHz}, \lambda_{z}=0.3$ in.

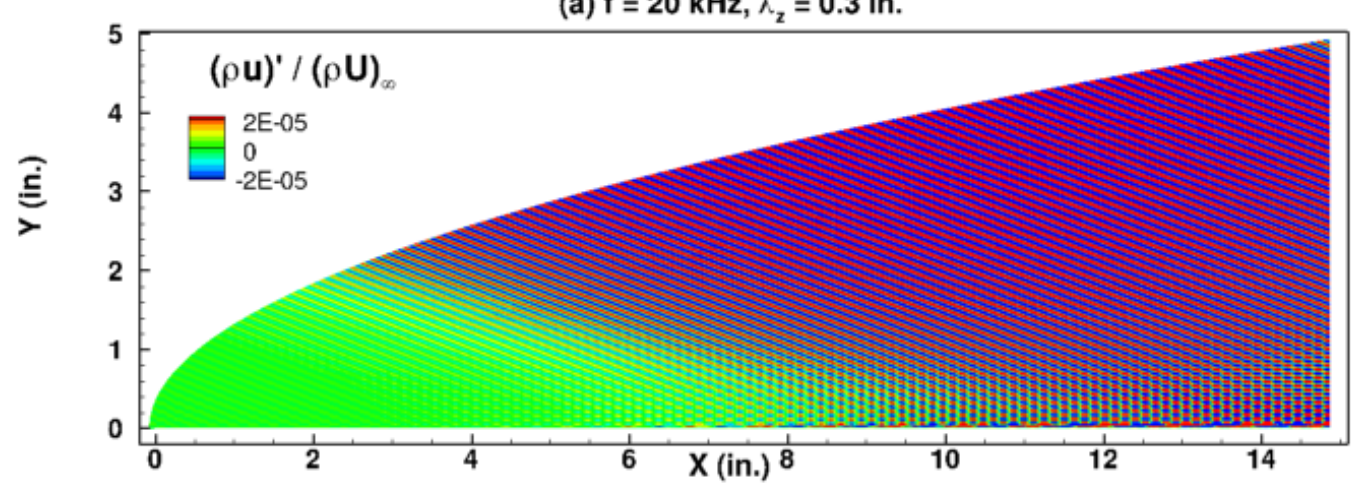

(b)

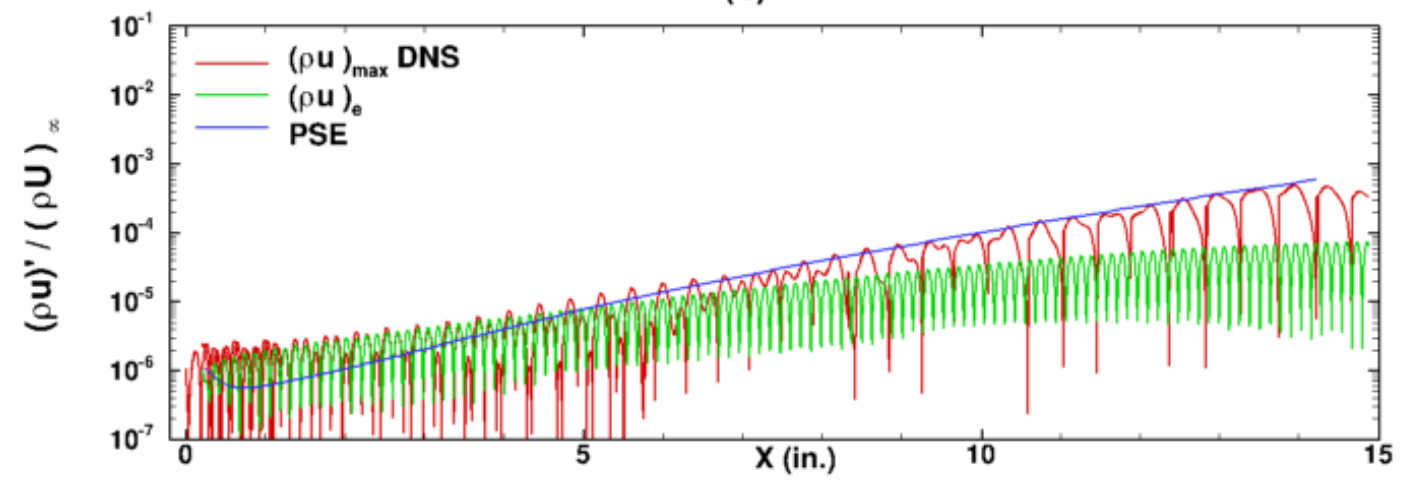

Figure 16. Fluctuations generated by the interaction of a nonuniform three-dimensional plane acoustic wave incident at 68 degrees with a flat plate. $f=20 \mathrm{kHz}, \lambda_{z}=0.3 \mathrm{in}, \theta_{y}=68.0 \mathrm{deg}$. (a) mass-flux contours at a fixed time, and (b) maximum mass-flux fluctuations inside and outside the boundary layer.

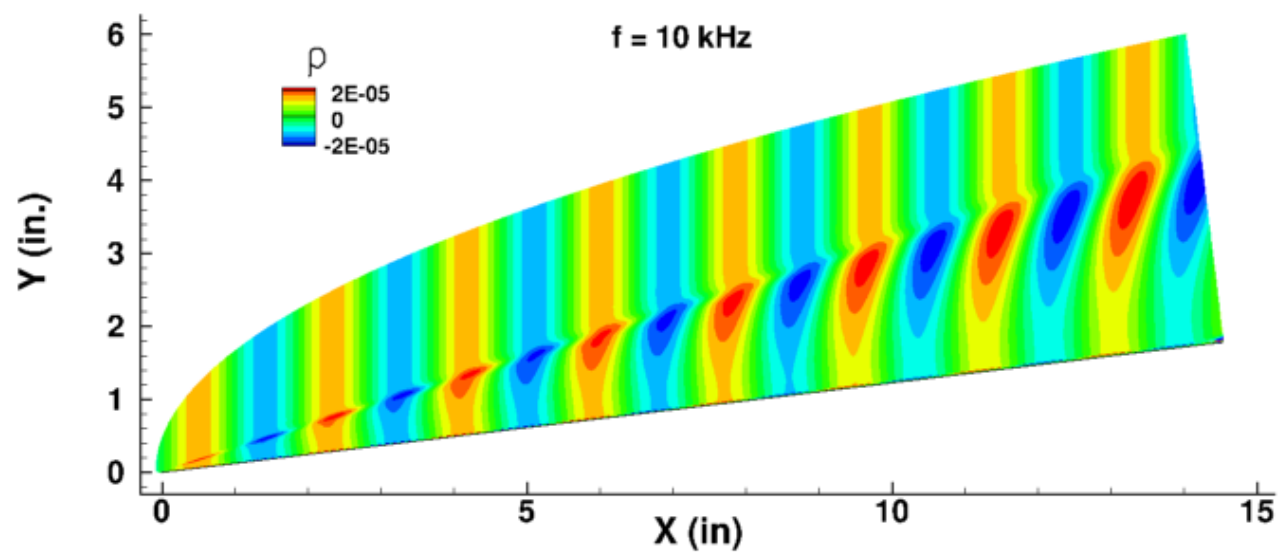

Figure 17. Contours of the unsteady density fluctuations generated by a two-dimensional uniform plane wave on a cone. $f=10 \mathrm{kHz}$. 


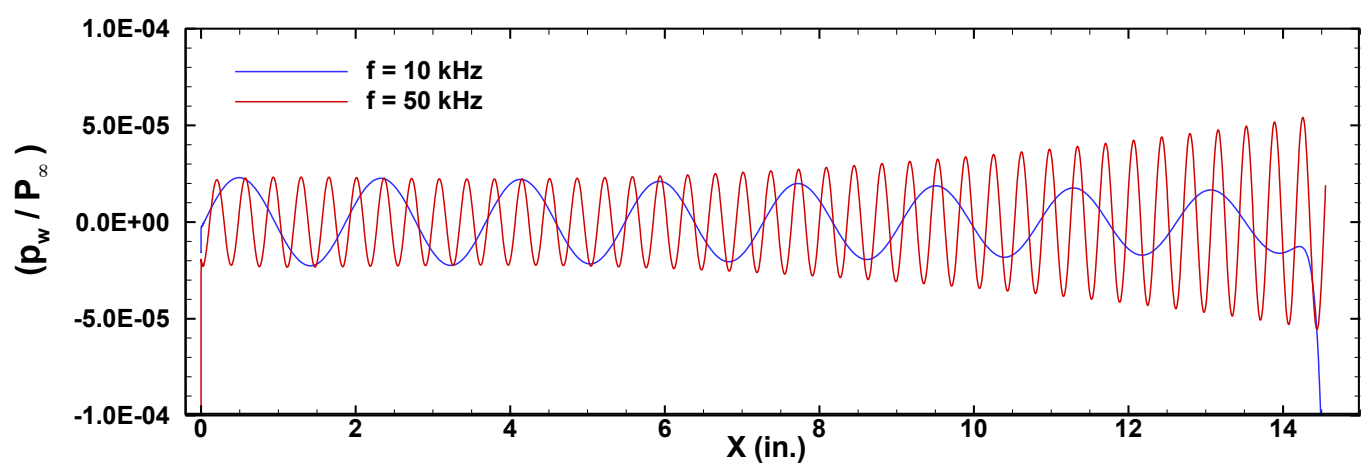

Figure 18. Wall pressure fluctuations on a cone generated by the two-dimensional uniform plane wave for the frequencies $f=10$ and $50 \mathrm{kHz}$.

(a) $f=10 \mathrm{kHz}, \mathrm{m}=0$

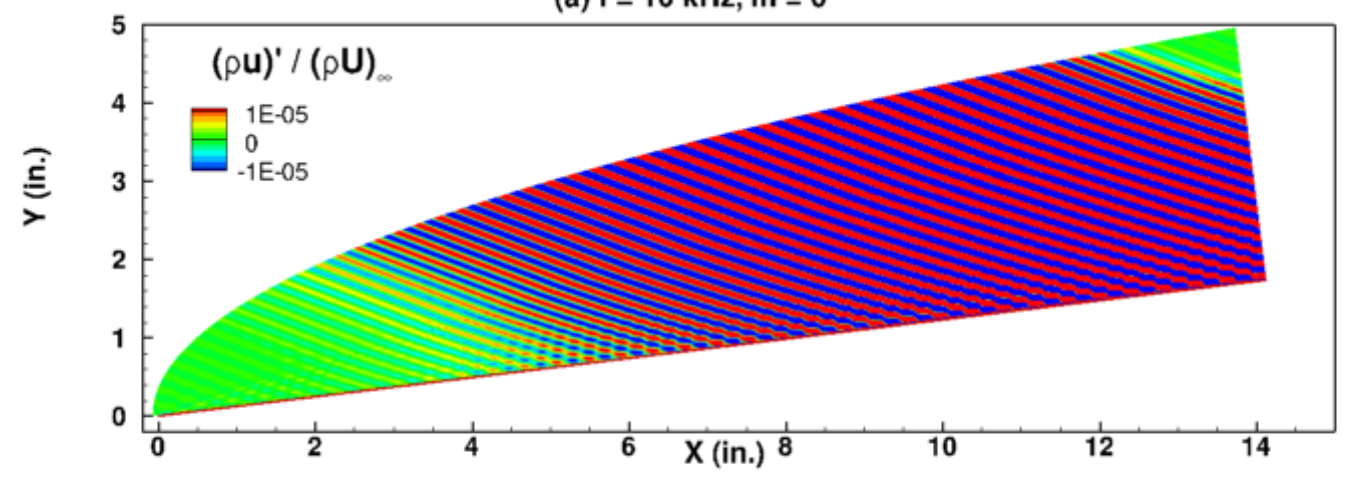

(b)

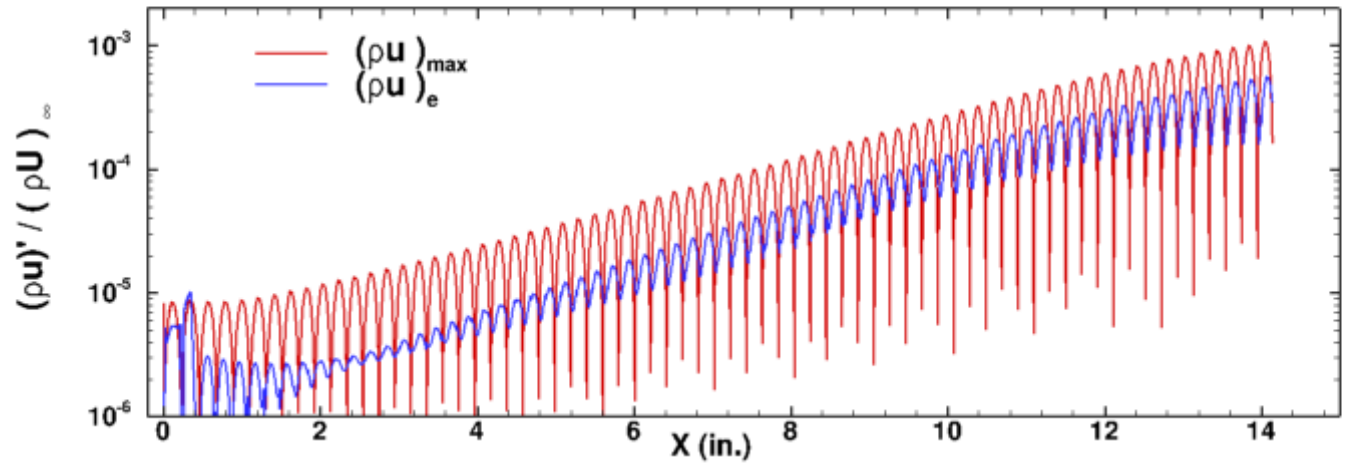

Figure 19. (a) The density contours at a fixed time and (b) maximum mass-flux fluctuations inside the boundary layer and the mass-flux fluctuations at the edge of the boundary layer generated by the interaction of acoustic field radiated from the nozzle wall and a cone obtained from DNS. 


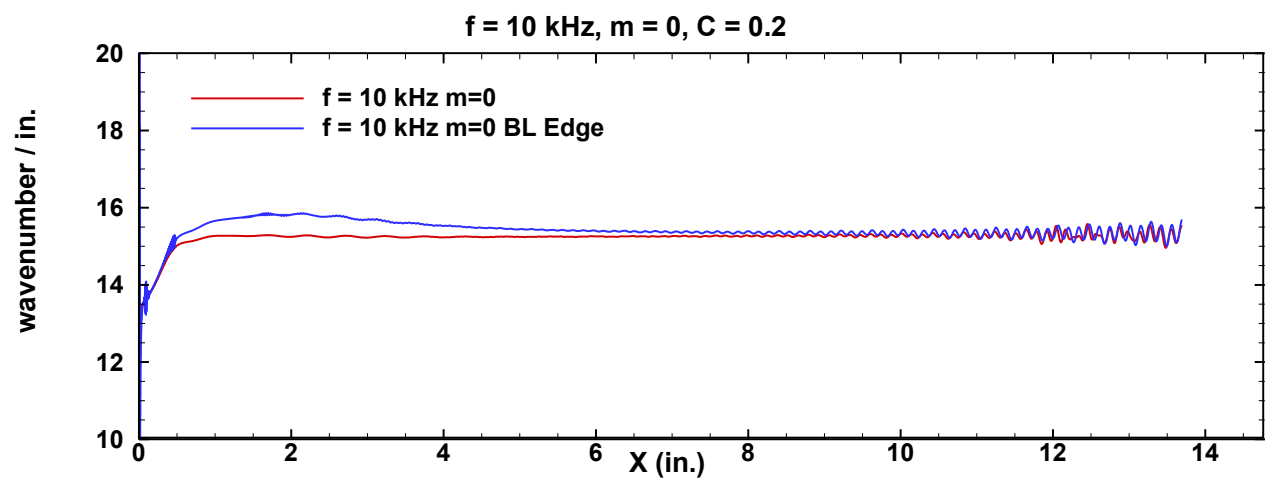

Figure 20. The variation of the wavenumber of the wall pressure versus boundary-layer edge fluctuationson the cone for $f=10 \mathrm{kHz}$ and $m=0$.

(a)

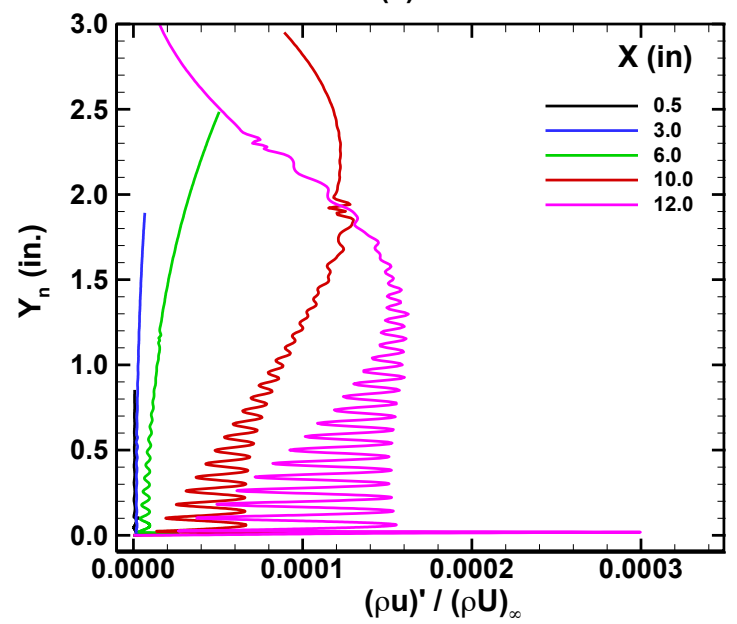

(b)

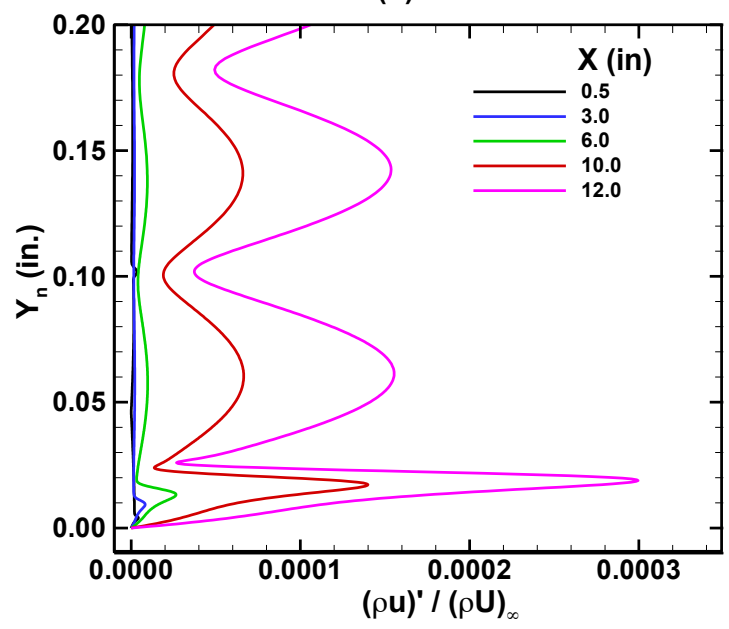

Figure 21. The amplitude of the mass-flux fluctuations at different axial locations, (a) inside and outside the cone boundary layer, (b) inside the boundary layer. $f=10 \mathrm{kHz}, m=0$.

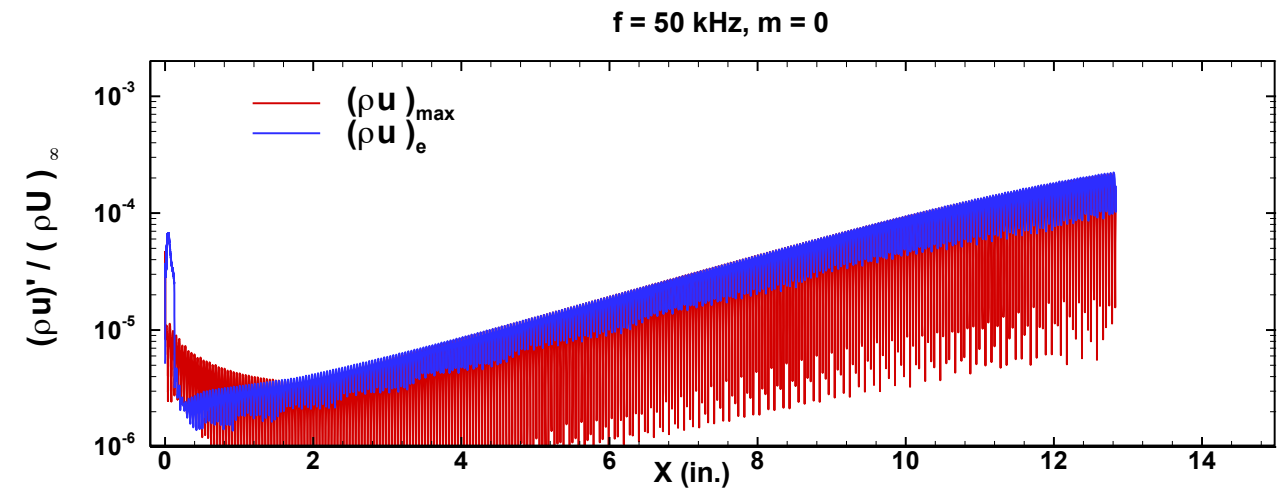

Figure 22. The maximum mass-flux fluctuations inside the boundary layer and the mass-flux fluctuations at the edge of the boundary layer generated by the interaction of acoustic field radiated from the nozzle wall and cone obtained from DNS. $f=50 \mathrm{kHz}, m=0$. 
(a)

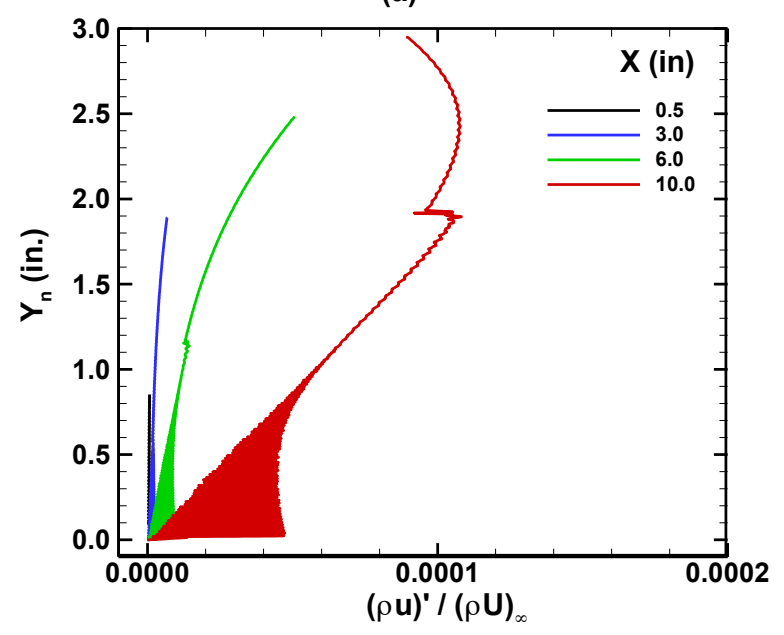

(b)

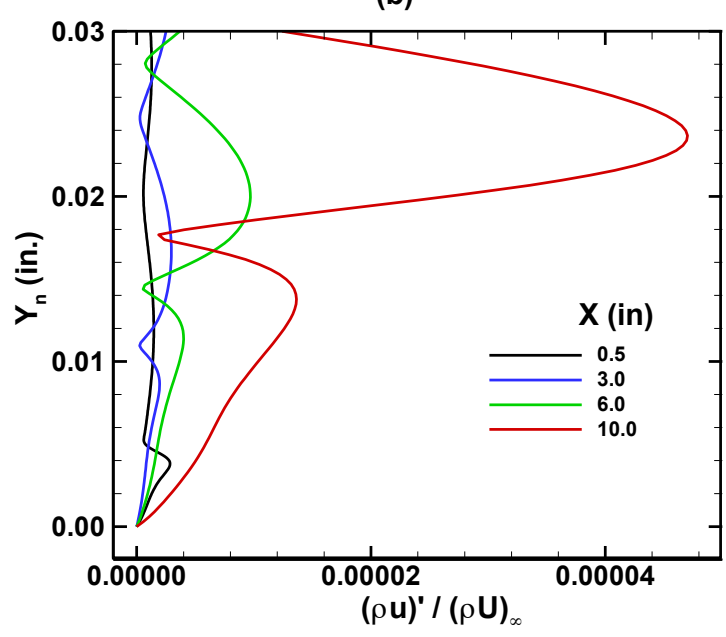

Figure 23. The amplitude of the mass-flux fluctuations at different axial locations, (a) inside and outside the cone boundary layer, (b) inside the boundary layer. $f=50 \mathrm{kHz}, m=0$.

(a) $\mathrm{f}=10 \mathrm{kHz}, \mathrm{m}=\mathbf{3 0}$

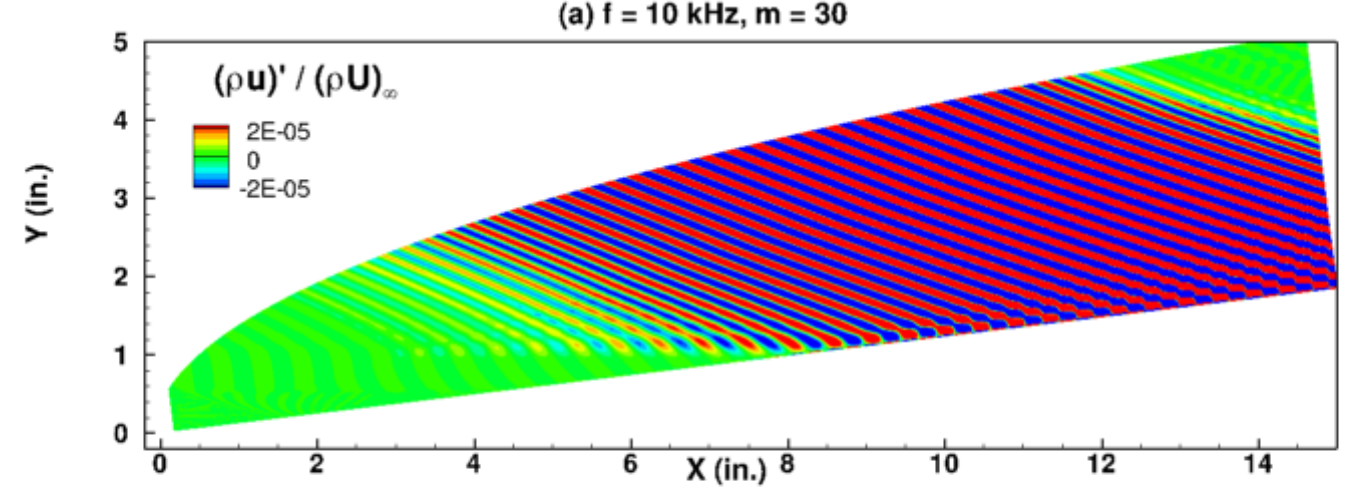

(b)

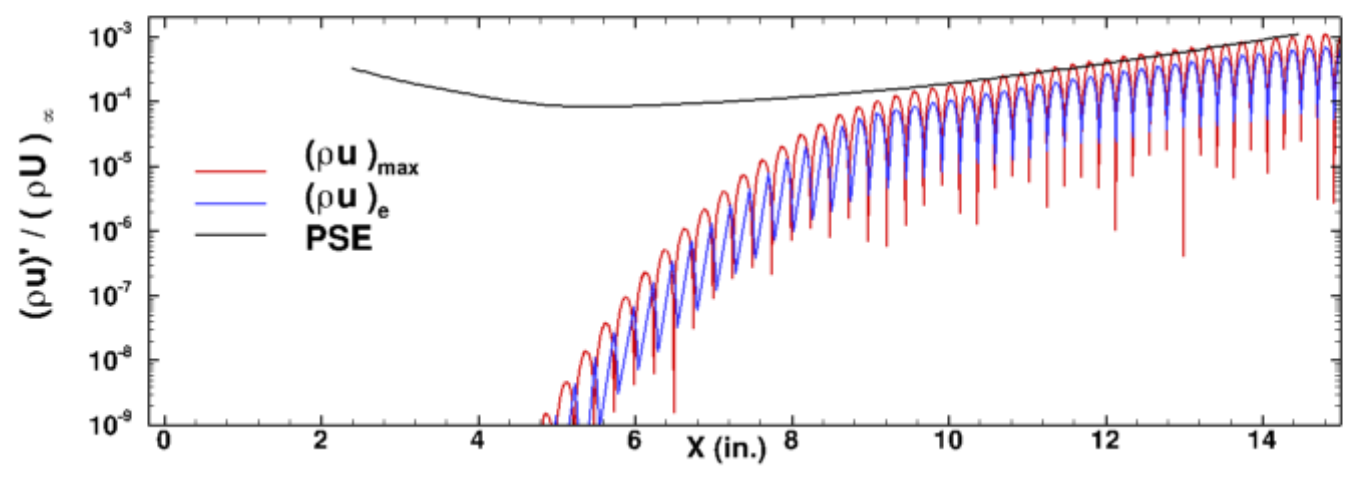

Figure 24. (a) The density contours at a fixed time and (b) maximum mass-flux fluctuations inside the boundary layer and the mass-flux fluctuations at the edge of the boundary layer generated by the interaction of acoustic field radiated from the nozzle wall and a cone obtained from DNS. $f=10 \mathrm{kHz}, m=30$. 


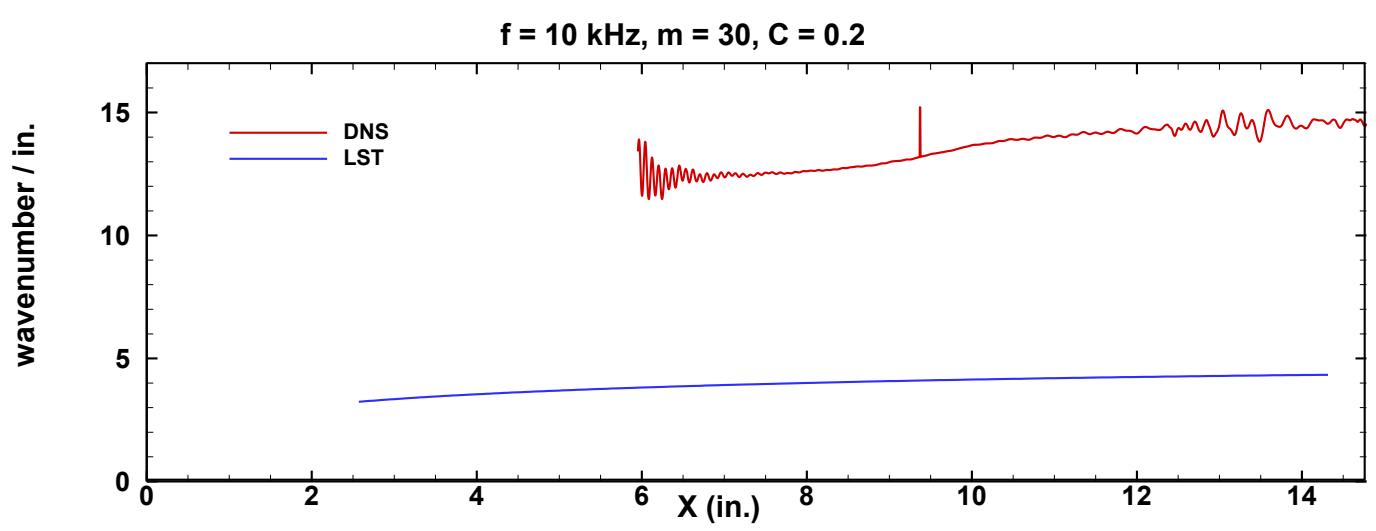

Figure 25. The variation of the wavenumber of the wall pressure fluctuations and LST computations on a cone. $f=10 \mathrm{kHz}, m=30$.

(a) $f=10 \mathrm{kHz}, \mathrm{m}=10$

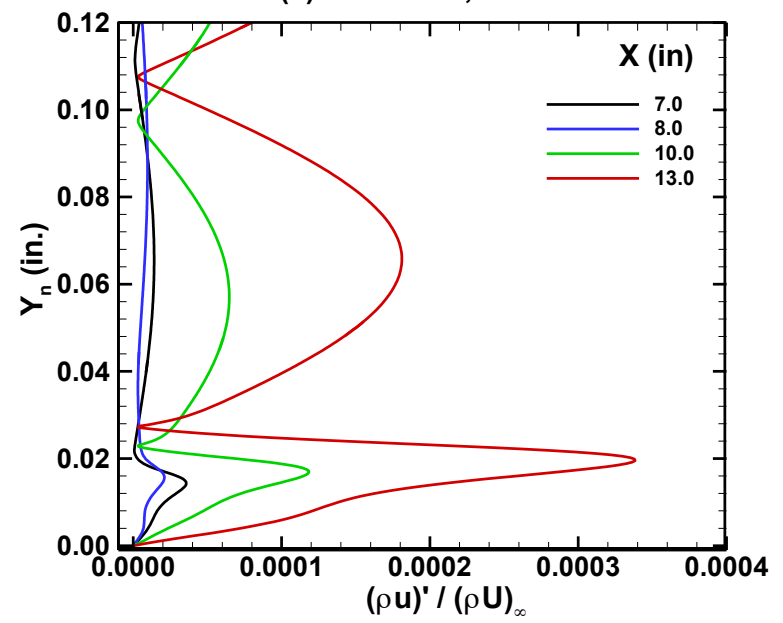

(b) $f=10 \mathrm{kHz}, \mathrm{m}=\mathbf{3 0}$

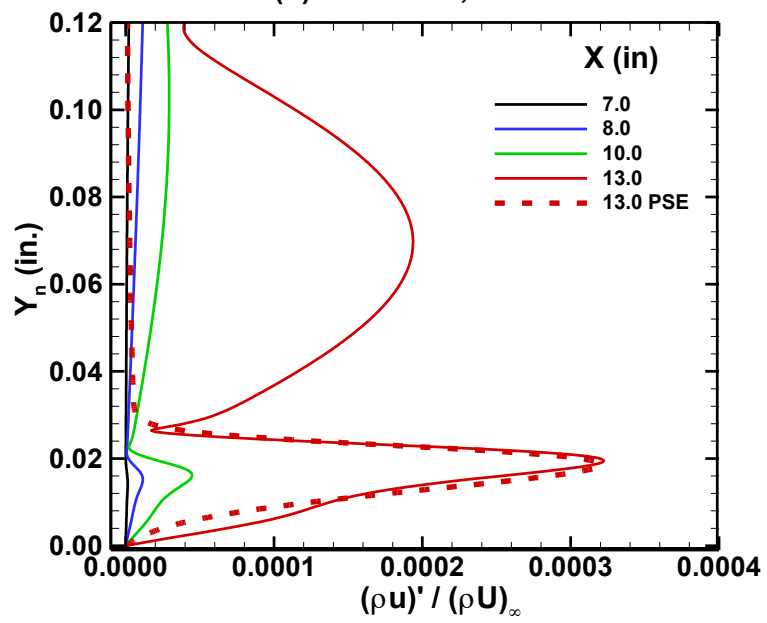

Figure 26. The amplitude of the mass-flux fluctuations at different axial locations on a cone for the frequency $f=10 \mathrm{kHz}$, (a) $m=10$, and (b) $m=30$.

$f=50 \mathbf{k H z}, \mathrm{m}=\mathbf{3 0}$

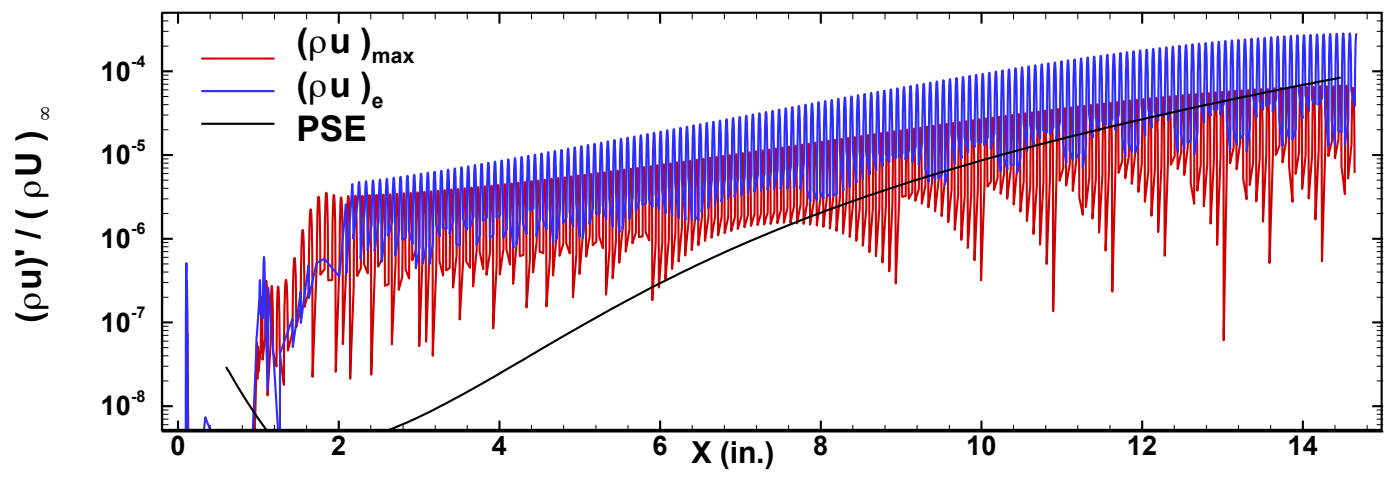

Figure 27. The maximum mass-flux fluctuations inside the boundary layer and the mass-flux fluctuations at the edge of the boundary layer generated by the interaction of acoustic field radiated from the nozzle wall and a cone obtained from DNS. $f=50 \mathrm{kHz}, m=30$. 
(a) $f=50 \mathrm{kHz}, \mathrm{m}=\mathbf{1 0}$

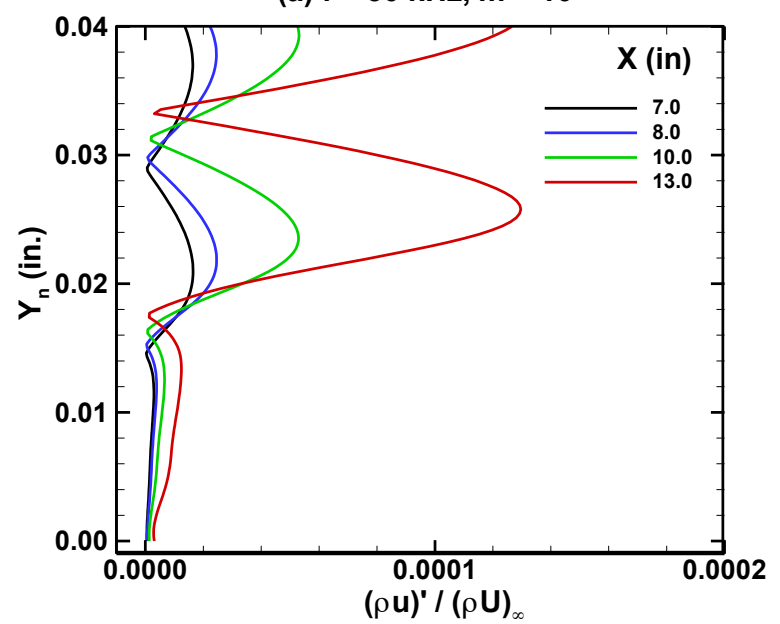

(b) $f=\mathbf{5 0 ~ k H z , ~} m=\mathbf{3 0}$

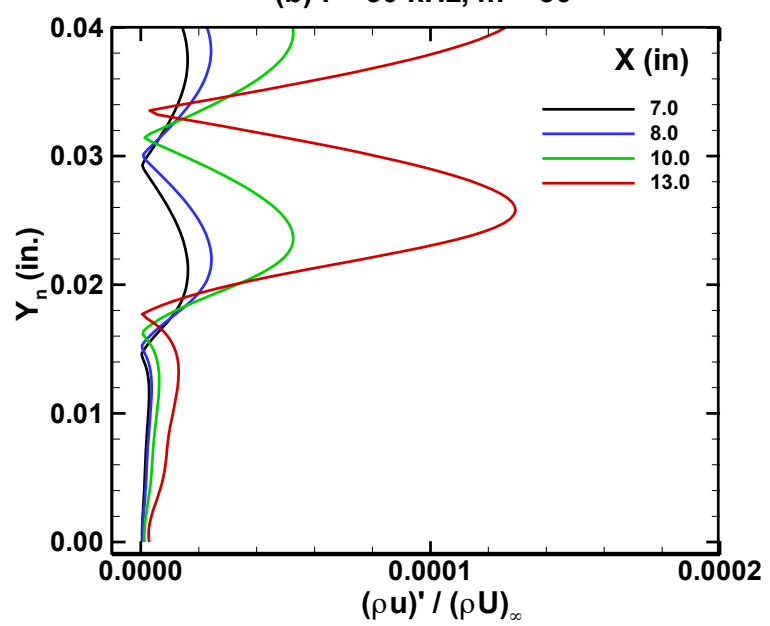

Figure 28. The amplitude of the mass-flux fluctuations at different axial locations on a cone for the frequency $f=50 \mathrm{kHz}$, (a) $m=10$, and (b) $m=30$.

(a) $\mathrm{f}=\mathbf{5 0} \mathrm{kHz}, \mathrm{m}=\mathbf{2 0}$

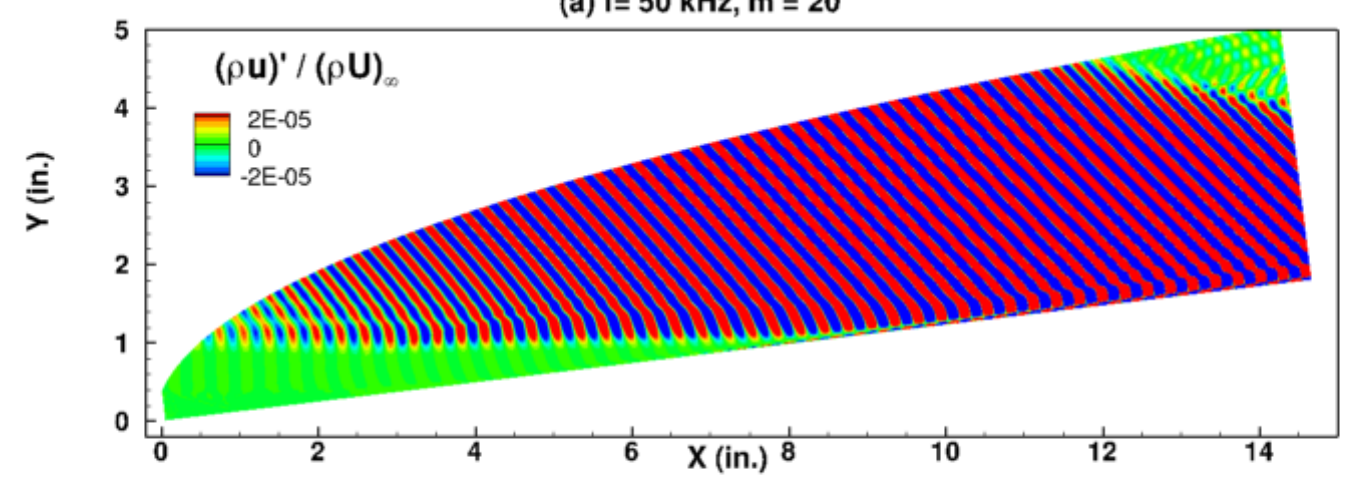

(b)

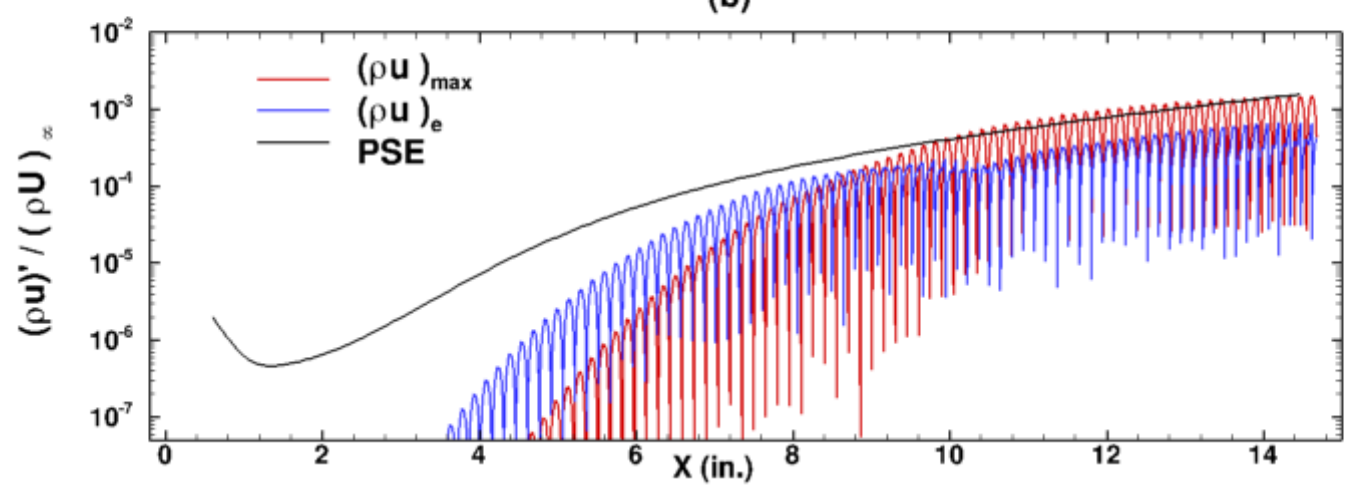

Figure 29. (a) The mass-flux contours at a fixed time and (b) maximum mass-flux fluctuations inside the boundary layer and the mass-flux fluctuations at the edge of the boundary layer generated by the interaction of acoustic field radiated from the nozzle wall and a cone obtained from DNS. $f=50 \mathrm{kHz}, m=20$. 


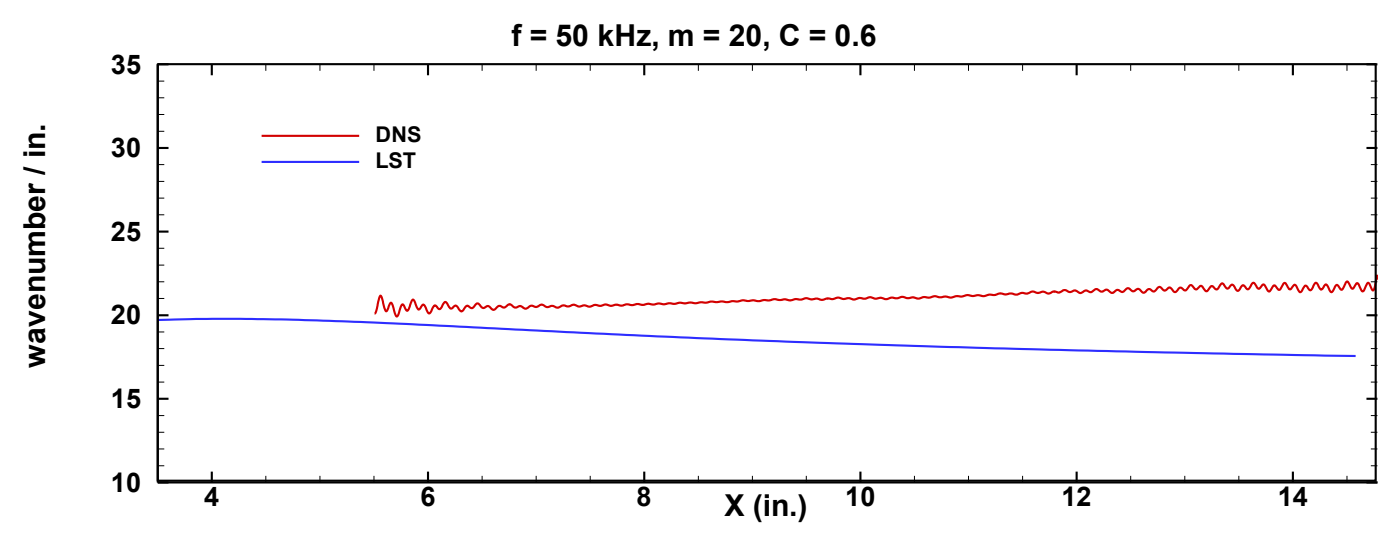

Figure 30. The variation of the wavenumber of the wall pressure fluctuations on a cone. $f=50 \mathrm{kHz}, m=20$.

(a) $\mathrm{f}=50 \mathrm{kHz}, \mathrm{m}=\mathbf{2 0}, \mathrm{C}=0.6$

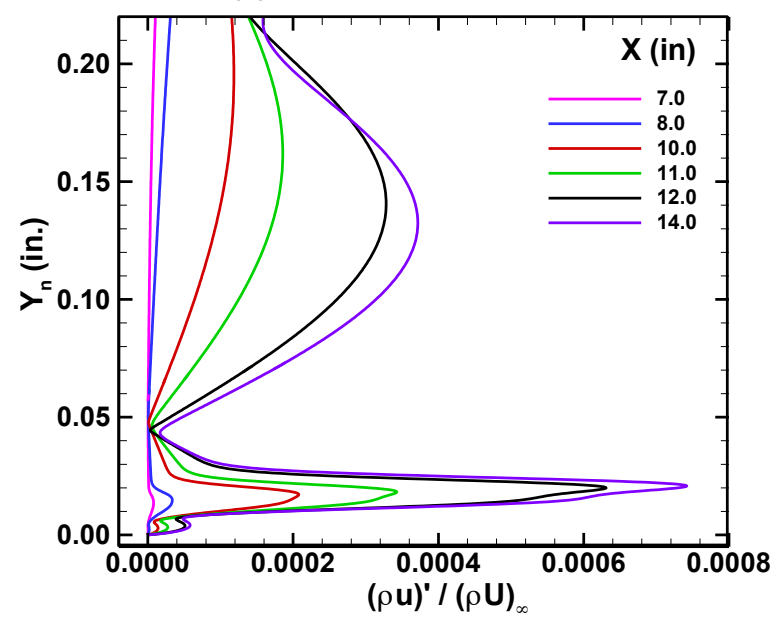

(b) $f=50 \mathrm{kHz}, \mathrm{m}=20, \mathrm{C}=0.6$

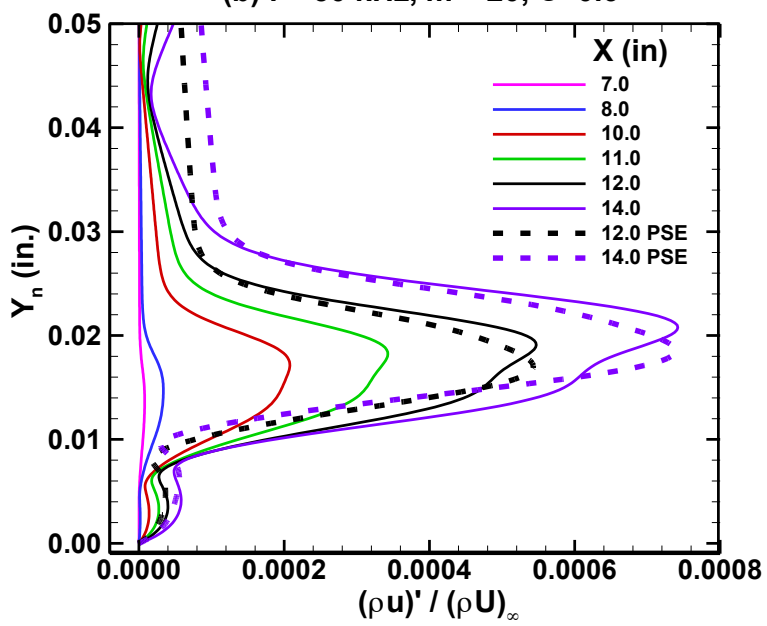

Figure 31. The amplitude of the mass-flux fluctuations at different axial locations, (a) inside and outside the boundary layer on a cone, (b) inside the boundary layer. $f=50 \mathrm{kHz}, m=20, C=0.6$. 